\title{
Trivium
}

Revue franco-allemande de sciences humaines et sociales - Deutsch-französische Zeitschrift für Geistesund Sozialwissenschaften

$7 \mid 2010$

Max Weber et la bureaucratie

\section{État moderne et bureaucratie moderne chez Max} Weber

\section{Hubert Treiber}

Traducteur : Olivier Mannoni

\section{CpenEdition}

Journals

Édition électronique

URL : http://journals.openedition.org/trivium/3831

DOI : $10.4000 /$ trivium.3831

ISSN : 1963-1820

Éditeur

Les éditions de la Maison des sciences de l'Homme

Référence électronique

Hubert Treiber, «État moderne et bureaucratie moderne chez Max Weber », Trivium [En ligne], 7 | 2010, mis en ligne le 02 mars 2016, consulté le 21 décembre 2020. URL : http://journals.openedition.org/ trivium/3831; DOI : https://doi.org/10.4000/trivium.3831

Ce document a été généré automatiquement le 21 décembre 2020.

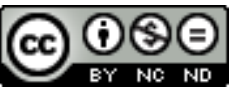

Les contenus des la revue Trivium sont mis à disposition selon les termes de la Licence Creative Commons Attribution - Pas d'Utilisation Commerciale - Pas de Modification 4.0 International. 


\title{
État moderne et bureaucratie moderne chez Max Weber
}

\author{
Hubert Treiber \\ Traduction : Olivier Mannoni
}

\section{NOTE DE L'ÉDITEUR}

Nous remercions Hubert Treiber de nous avoir accordé l'autorisation de traduire cet article dans le présent numéro. La présente traduction a été révisée en janvier 2016.

Moderner Staat und moderne Bürokratie bei Max Weber

\section{I.}

1 Pour représenter les liens réciproques qui unissent l'État et la bureaucratie modernes, il semble que nous puissions prendre comme point de départ cette définition de l'État datant de la dernière période de l'existence et du travail de Weber, que l'on peut lire dans les Concepts fondamentaux de la sociologie : « Nous entendons par État une entreprise politique à caractère institutionnel [politischer Anstaltsbetrieb] lorsque et tant que sa direction administrative revendique avec succès, dans l'application des règlements, le monopole de la contrainte physique légitime ${ }^{1}$.»

2 Plusieurs raisons plaident en faveur du choix de cette définition; la principale est le fait que les caractéristiques qui y sont exposées ne permettent pas seulement d'étudier les principales dimensions du concept wébérien de l'État ${ }^{2}$, mais aussi et simultanément d'établir un lien étroit avec la bureaucratie (direction administrative). C'est surtout la caractéristique de l'entreprise à caractère institutionnel ou de l'institution [Anstalt] (à laquelle Weber accorde une attention particulière dans son texte sur les catégories ${ }^{3}$, dans sa sociologie du droit ${ }^{4}$ et dans les Concepts fondamentaux de la sociologie $e^{5}$ qui justifie le choix en faveur de la définition de l'État que nous avons citée ${ }^{6}$. D'une part, le concept d'institution, avec la caractéristique des ordres statutaires imposés ${ }^{7}$, se révèle comme 
point final d'une évolution que Weber a résumée en une phrase dans la Sociologie $d u$ $d r^{8} t^{8}$, évolution qui, dans le $\$ 2$ de la Sociologie du droit, est aussi désignée comme un processus juridique de rationalisation, ce qui associe la définition de l'État citée plus haut au théorème de la rationalisation chez Weber. D'autre part, en faisant appel à un concept juridique exact pour l'examen sociologique, Weber met en œuvre l'annonce qu'il avait faite d'un point de vue méthodologique et selon laquelle « au cours de l'étude de l'activité réelle qui manifeste partout de constantes transitions entre les cas "typiques" ", la sociologie doit inévitablement "très souvent utiliser les expressions rigoureuses (parce que fondées sur une interprétation syllogistique des normes) du droit, quitte à leur substituer par la suite son propre sens, radicalement différent du sens juridique ${ }^{9} »$. Mais c'est précisément là que le concept sociologique d'institution (dans les Concepts fondamentaux de la sociologie comme dans l'essai sur les catégories de ce que l'on appelle la Théorie de la science) met en avant ces caractéristiques propres au concept d'institution dans les sciences du droit, ce qui pose de singulières difficultés à un mode d'observation sociologique orienté vers «l'État » pour laquelle l'expression "État» ne désigne que le «déroulement d'une activité humaine d'une espèce particulière ${ }^{10}$ ", même si la notion sociologique d'institution - au sens de l'existence «de règlements rationnels établis par des hommes et [d']un appareil de contrainte conçu comme un élément contribuant à déterminer l'activité ${ }^{11} »-$ ne néglige pas totalement le niveau de l'action. Si cette dimension du niveau de l'action avait été intégrée à la réflexion, la réception du modèle wébérien de la bureaucratie par la sociologie de l'organisation américaine (mais aussi par les représentants de l'enseignement de la gestion en Allemagne) aurait été différente ${ }^{12}$. Sur le plan conceptuel et systématique, cela a été interprété de manière convaincante comme un rétrécissement de la vision ${ }^{13}$ - la proximité du concept wébérien d'institution avec la théorie de l'institution de l'État sur la ligne de l'école Gerber / Laband, comme avec la théorie de l'institution relevant du droit public chez Otto Mayer, avec une conception de l'État autoritaire et monarchique, centrée sur le pouvoir ${ }^{14}$, influence de manière non négligeable la conception que Weber a de l'État. Cela, se révèle donc avantageux dans la mesure où cette conception liée au pouvoir fait apparaître l'appareil bureaucratique comme une dimension de la sociologie de l'État. À cette restriction du regard au niveau conceptuel et systématique, due à un emprunt à la terminologie juridique ${ }^{15}$, on peut cependant opposer un élargissement de la vision lourd de conséquences, dû à la volonté de Weber de ramener des entités collectives comme «l'État» au niveau de l'activité d'individus, activité orientée par un sens, en tenant compte du fait que l'on associe à ce type de structures collectives des représentations très précises que les individus prennent comme points de repère et auxquelles ils réagissent, le cas échéant, par un comportement adéquat. Si l'on suit ce mode d'observation de Weber (qu'il faut toujours intégrer à la réflexion) qui conçoit « l'État » comme un « complexe spécifique d'activité commune accomplie par des hommes ${ }^{16} »$, alors les conceptions postmodernes qui voient dans "l'État» une "structure polycentrique» ou le font dissoudre dans des réseaux politiques se rapprochent de manière extrêmement surprenante de Weber, ce Weber dont les conceptions sur la «bureaucratie moderne» sont qualifiées de «dépassées» ou ne correspondant plus à des conditions d'environnement transformées ${ }^{17}$. 


\section{II.}

Nous présenterons brièvement dans ce chapitre les principales caractéristiques de la définition que nous avons exposée en introduction, en nous efforçant tout particulièrement de faire apparaitre les liens qui rattachent ces grands traits mais aussi d'arriver à une présentation de la bureaucratie comme forme de domination spécifiquement moderne, dont la genèse ne découle pas seulement de certaines conditions déterminées ${ }^{18}$ mais constitue aussi pour Weber "le germe de l'État occidental moderne ${ }^{19}$ ».

4 (1) Lorsque Weber décrit l'État comme une "entreprise institutionnelle politique ", l'association des mots «institution » et «entreprise » vise dans un premier temps la durée et la continuité ${ }^{20}$; simultanément, le caractère d'entreprise de l'État moderne renvoie au fait qu'«au fond, sa nature est parfaitement identique » à celle de la fabrique et, pour ce qui concerne le « rapport de domination » existant dans chaque cas "au sein de l'entreprise», présente un autre point commun structurel dans la «concentration des moyens objectifs de l'entreprise» entre les mains de l'entrepreneur ou du «maître politique ${ }^{21}$ ». Pour caractériser plus précisément la notion d'institution, dont les caractères, nous l'avons déjà signalé, sont empruntés à l'économie conceptuelle juridique de cette époque, on fait appel aux éléments de définition, pas tout à fait identiques, présentés dans l'essai sur les catégories et dans les Concepts fondamentaux de la sociologie ${ }^{22}$. L'institution, en tant que forme de societatisation constituée sous forme d'action, se distingue - selon les principes des Concepts fondamentaux - par une cohésion régulée (vers l'extérieur) de manière rationnelle, ainsi que par des ordres institués rationnellement qui sont imposés aux personnes soumises au pouvoir de l'institution ("participants contraints»), c'est-à-dire imposés «sur la base d'une "attente relative à une entente" 23 ". Le caractère de domination et de contrainte est certes déjà intégré au concept de "Oktroyierung" [le fait d'imposer un statut, $\mathrm{Ndlr}]^{24}$, mais ce n'est que dans la définition de l'institution développée dans l'essai sur les catégories que Weber le fait expressément. La définition a explicitement recours à la caractéristique de "l'appareil de contrainte ", et il note à son propos que celui-ci codéterminerait de fait l'action de ceux qui sont soumis au pouvoir qui impose un statut [Oktroyierung] $]^{25}$. L'attribut "politique " ajouté à l'entreprise institutionnelle renvoie lui aussi à l'aspect de la "violence» ou de la "domination", sans l'aide desquels on ne pourrait définir le « caractère politique d'un groupement ${ }^{26}$ ».

On a déjà attiré l'attention sur le fait que la caractéristique des règlements statutaires respectant une rationalité (avec finalité) caractérise aussi le point final d'une évolution à laquelle est soumis le théorème de rationalisation de Weber. Ainsi, les distinctions conceptuelles entreprises dans l'essai sur les catégories (activité communautaire, associative et sociale, d'un côté, action de groupement et d'institution de l'autre) ne constituent donc pas seulement une structure de relation logique, mais renvoient aussi simultanément à une relation qui évolue au cours de l'histoire et qui « exprime le degré spécifique du potentiel social de rationalisation qu'ils recèlent ${ }^{27}$ ».

6 (2) En nommant explicitement la direction administrative dans cette définition de l'État, Weber se rattache aux définitions du groupement et de la domination ${ }^{28}$, où il vise explicitement l'aspect de l'instauration par la force des ordres de groupement ainsi que le caractère de domination qui s'attache à une relation sociale fermée vers l'extérieur. Lorsqu'il attire précisément l'attention ${ }^{29}$ sur le fait que les membres de la direction 
administrative se distinguent par une attitude interne spécifique face à la « direction du groupement ", ce sont les hypothèses fondamentales de la sociologie wébérienne de la domination qui entrent en jeu. Il est facile de les découvrir si l'on considère les réflexions de Weber sur la sociologie de la domination comme une réponse à la question de Hume : «Why are the many governed by the few ?» On voit que la domination $\mathrm{du}$ petit nombre sur le grand nombre repose sur leur capacité supérieure d'organisation, qui consiste notamment dans l'édification d'une direction administrative et dans la aptitude à en disposer; mais elle tient aussi au fait qu'ils sont capables d'éveiller et de maintenir la foi dans la légitimité [Rechtmäßigkeit] de leur domination - en premier lieu auprès des membres de la direction administrative, mais aussi chez les dominés. S'il s'ajoute, au pilier extérieur d'une domination (c'est-à-dire à une direction administrative), des motifs de justification internes, celle-ci se révèle incomparablement plus stable qu'une domination qui n'est pas capable d'honorer sa prétention à la légitimité ${ }^{30}$.

(3) Avec la caractéristique, particulièrement mise en valeur, du monopole de la contrainte physique légitime ${ }^{31}$ pour la mise en œuvre des organisations, Weber souligne une nouvelle fois que "l'État moderne » (tout comme les groupements politiques ou les communautés politiques qui le précèdent historiquement ${ }^{32}$ ) est un «rapport de domination de l'homme sur l'homme» étayé sur le moyen "de la violence [Gewaltsamkeit] ${ }^{33}$ ", ceux qui sont soumis à la domination considérant l'utilisation de la violence comme «légitime ${ }^{34}$ ». Il faut appeler, avec Hermes ${ }^{35}$, que Weber ramène au charisme militaire le monopole (légitime) de la violence et la compétence (légitime) à édicter des statuts ${ }^{36}$. Lorsque tous deux - monopole de la violence et capacité d'établir un statut - sont donnés, le groupement politique devient État, qui garantit la validité de ses organisations statutaires en mettant en œuvre la violence légitime. Cela renvoie simultanément à la fonction ${ }^{37}$ créatrice d'ordre exercée par la violence, de la même manière que tout ordre social engendré par la force a besoin de la force pour limiter sa force. "Tout projet d'ordre est soumis à ce cercle vicieux de la maîtrise de la force : l'organisation sociale est une condition nécessaire de l'endiguement de la force - la force est une condition nécessaire du maintien de l'organisation sociale ${ }^{38}$.» Pour l'État moderne, il est aussi caractéristique, dans le contexte de la question que nous venons de soulever à propos des limites que l'on doit apporter à la force institutionnalisée, qu'il n'existe de "violence "légitime" que dans la mesure où l'ordre étatique la permet ou la prescrit ${ }^{39}$ ». L'État moderne est ainsi un État de droit, ce que Weber souligne par la formule selon laquelle "aujourd'hui, la contrainte juridique par la force est le monopole de l'institution étatique ${ }^{40}$ ». Puisque Weber parle de "droit» lorsque les ordres statutaires sont garantis de l'extérieur par la force ${ }^{41}$ (direction contraignante), et qu'il parle, en cas « d'existence factuelle d'une [...] force (de commandement), si les commandements de celle-ci sont aussi respectés dans les faits, d'une domination, qui, pour sa part, est en quête de légitimitét ${ }^{42}$, la domination et le droit sont, du point de vue catégoriel, liés l'un à l'autre par le biais de la légitimitét ${ }^{43}$. Si, en outre, le concept de domination ${ }^{44}$ 'a pas pour seul but le respect effectif d'un commandement, mais exige que les dominés fassent « du contenu du commandement, au nom du commandement lui-même, la maxime de leur activité » et l'acceptent comme "norme en vigueur », ce n'est pas seulement de cette manière une acceptation générale du rapport de domination ("petite légitimité »), qui est postulée, mais aussi une compréhension ${ }^{45}$, telle qu'elle s'exprime en particulier dans la notion de "représentation de la norme » dans l'essai sur Stammler ${ }^{46}$ et dans le « concept de validité » de l'essai (ou fragment de 
texte) Die Wirtschaft und die Ordnungen ${ }^{47}{ }^{48}$. Dans cette optique, les commandements indiquant l'exercice de la domination apparaissent comme des "maximes", dans le sens de « règles » considérées comme «obligatoires ou exemplaires » et qui font office de «motifs de détermination effectifs de l'activité humaine réelle». Selon Weber, conformément au concept de validité, y sont associés un «devoir » ou "l'idée d'une obligation » qui agit durablement sur ces « motifs de détermination » et augmente ainsi la probabilité d'orientation sur des "maximes que l'on peut expliciter» (sur des " ordres » statutaires ou sur le « droit») et, pour finir, sur leur respect ${ }^{49}$. On peut donc affirmer que «le groupement politique n'est pas seulement le domaine de validité empirique de commandements "acceptés" de manière normative (= domination) mais aussi le domaine d'action empirique de normes garanties par la contrainte $(=\mathrm{droit})^{50}$ ».

8 Comme toute direction administrative, la bureaucratie moderne se distingue par le fait "qu'un cercle de personnes [...] habitué à obéir aux ordres de chefs se tient en permanence à disposition et se répartit l'exercice de ces pouvoirs de commandement et de contrainte qui servent au maintien de la domination ("organisation") ${ }^{51} »$. Ce qui vaut pour les dominés s'applique tout particulièrement aux bureaucrates : s'ils obéissent aux ordres, c'est notamment parce que "la "validité" (de ce) pouvoir de commandement " s'inspire de l'idée de l'existence d'un ordre considéré comme "obligatoire et exemplaire » (« légitime ») c'est-à-dire, dans le cas de l'État moderne, d'un ordre (de droit) posé de manière statutaire et rationnelle, et auquel sont aussi soumis les dominants eux-mêmes. Cela apparaît aussi dans l'activité administrative des bureaucrates qui satisfont au "devoir objectif de leur fonction" et " "sans considération de personne" exercent de manière rigoureusement formaliste, en fonction de règles rationnelles - et, lorsque celles-ci font défaut, en fonction de points de vue liés à la fonctionnalité concrète ${ }^{52}$ »-les affaires liées à leurs fonctions. Pour cette raison, dans le quotidien de l'administration, pour autant que celle-ci se rapproche du type de la domination légale ${ }^{53}$, la violence, comme moyen de domination, est remplacée par le procédé juridique, bureaucratique, et devient par là-même « invisible ${ }^{54}$ ».

Dans sa critique d'une lecture, représentée par Renate Mayntz $z^{55}$, du type de bureaucratie wébérien, Tyrell indique "que l'activité bureaucratique en tant que telle, dès lors qu'elle est, en premier lieu, "programmée sous forme conditionnelle", n'est typiquement pas une activité rationnelle en finalité [zweckrational $]^{56} »$. La programmation conditionnelle, dans l'esprit d'une succession de conditions et de conséquences, n'est pas seulement le code résumant la succession de l'ordre et de l'obéissance ${ }^{57}$, mais aussi la succession prescrite de l'état de fait et de la conséquence juridique. C'est pourquoi Weber utilise, et ce n'est pas par hasard, l'image de "l'automate du droit ", qui s'applique certes au juge, mais est également utilisable pour le fonctionnaire de l'administration, automate «dans lequel on insère, en haut, le fait et ses coûts, afin qu'il recrache en bas le verdict et les motifs ${ }^{58}$ ". L'image de distributeur de droit ou d'articles de lois fait penser aux juristes de l'administration formés à l'université, tel que les a notamment produits l'Allemagne ${ }^{59}$. Elle vise aussi le "spécialiste humainement impartial, et donc rigoureusement "objectif" 60 " qui décide selon des "règles calculables" universelles et qu'il faut ainsi considérer interchangeable par principe, parce que n'importe quel fonctionnaire formé dans sa discipline fonctionne de la même manière, d'autant plus que le formalisme s'inscrit «dans l'essence de toute formation juridique, y compris celle des juges et des fonctionnaires d'administration, si l'on ne veut pas cultiver l'arbitraire ${ }^{61}$ ». L'image du distributeur de paraphes, appliqué à la bureaucratie moderne, se rapporte toutefois 
aussi au « "fonctionnement" précis, univoque, linéaire, toujours lié à des règles et donc prévisible de l'exécution bureaucratique des [...] affaires administratives ${ }^{62}$; c'est la raison pour laquelle Weber conserve aussi l'ancienne métaphore de la machine ${ }^{63}$ ou de la mécanisation ${ }^{64}$. La mécanisation, dans ce contexte, renvoie moins à «l'uniformité de l'activité commandée " ${ }^{65}$ qu'au "dressage mécanisé et à l'insertion de l'individu dans un mécanisme auquel il ne peut échapper et qui le force à "suivre" 66 » à la fois en tant que cause et conséquence de la discipline, dont, aux yeux de Weber, la bureaucratie passe pour "l'enfant le plus rationnel ", notamment en raison de son " "objectivité" imperturbable », ce qui la rend en même temps vulnérable à la tentation de se mettre "à la disposition de tout pouvoir» qui réclame son service ${ }^{67}$. À l'inverse, la machine bureaucratique, comme n'importe quelle autre, engendre également des contraintes d'adaptation d'une nature particulière, aussi bien chez les bureaucrates eux-mêmes que chez ceux qui dépendent de leurs prestations, notamment dans le domaine de la prévoyance. Ceci est aussi favorisé par « l'inclination des fonctionnaires à traiter dans un sens matériel et utilitaire les tâches d'administration qui sont les leurs au service du bonheur des administrés ${ }^{68}$ ", tâches qui répondent aux revendications des catégories défavorisées. D’une manière générale, la bureaucratie peut perdre son caractère instrumental de «servante de la politique » et gagner en autonomie, dans la mesure où elle parvient à se soustraire à la prétention de la politique à diriger, en s'appuyant sur ses connaissances spécialisées et fonctionnelles par principe supérieures ${ }^{69}$. Cela dit, la bureaucratie moderne se distingue justement par une série de mesures préventives qui visent toutes à assurer son caractère instrumental : les fonctionnaires sont ainsi avant tout coupés des moyens d'administration et ne peuvent prétendre à être propriétaires de leurs fonctions. Il faut toujours se rappeler que Weber adopte une attitude ambivalente à l'égard de la bureaucratie moderne. D'une part, il accorde à la domination rationnelle légale (dont la bureaucratie serait le type le plus pur) la capacité «de principe de transformer et de varier ses règles». Cette aptitude place l'État moderne en situation de maintenir l'appareil bureaucratique flexible et adaptable dans des conditions générales en changement - ce qui est du reste aussi l'une des conditions pour que l'État en formation puisse se libérer des liens du traditionalisme ${ }^{70}$. D'autre part, Weber parle également des stratégies opposées et inspirées d'un "utilitarisme matériel » des bureaucrates, visant à défendre leurs acquis et à refuser d'apprendre, dont de nombreuses études empiriques sur la bureaucratie ont depuis démontré l'existence "dans une prétendue réfutation de Weber ${ }^{71}$ ». La revalorisation de la rationalité matérielle que l'on peut observer en même temps est à la fois la cause et la conséquence d'une autonomisation de la bureaucratie, qui - dans la mesure où sa rationalité formelle s'absolutise - retombe dans des modèles pré-rationnels ${ }^{72}$. Cela ne semble donc pas être un hasard si la bureaucratie de Kafka, qui présente ce type de traits «absolutistes", répond ne serait-ce que pour cette raison aux caractéristiques traditionnelles de la bureaucratie patrimoniale ${ }^{73}$ ou si des organisations bureaucratiques (administration des cadres) ${ }^{74}$ de l'ancien bloc de l'Est ont plutôt été considérées à la lumière du type de la bureaucratie traditionnelle ${ }^{75}$. L'image kafkaïenne de la bureaucratie comme appareil technique ayant tendance à « intégrer toujours plus largement des aspects de notre existence autrefois [...] gérés de manière libre et naturelle, à les aspirer dans ses chambres, casiers et sous-casiers ${ }^{76}$ » est facilement compatible avec les visions qu'avaient Alfred et Max Weber d'une machine bureaucratique que l'on croit capable, dans certaines conditions, "de fabriquer l'habitacle de [la] servitude des temps futurs ${ }^{77}$ ». Pareille " égyptisation de la société » 
menée de manière rationnelle semble pour Weber se situer dans le domaine du possible dans la mesure où pour les sujets devenus "hommes d'ordre ${ }^{78}$ ", " une administration aux mains de fonctionnaires, une administration et une intendance bonnes du point de vue technique, autrement dit rationnelles, [sera] l'unique et ultime valeur [qui] décidera de la manière dont leurs affaires doivent être menées ${ }^{79} »$.

Outre les conséquences de la bureaucratisation que nous venons brièvement d'aborder, Weber traite avant tout des conditions de sa genèse et de ses tendances à la propagation. La bureaucratie comme phénomène moderne est apparue en Occident en même temps qu'une économie monétaire et qu'un capitalisme en formation ${ }^{80}$, lequel avait intérêt à obtenir la sécurité du droit et la prévisibilité, deux qualités qui favorisent à leur tour ce capitalisme et que la rationalité formelle de la bureaucratie et du système de droit (qui lui correspond) est le mieux à même de garantir ${ }^{81}$. La création d'armées permanentes (sur le continent) ${ }^{82}$ et l'administration fiscale nécessaire à leur financement ${ }^{83}$ ont tenu une part considérable dans la construction et l'extension d'un appareil moderne d'administration et de fonctionnaires ${ }^{84}$, une technique avancée dans le domaine de l'information et des transports et, d'une manière générale, l'extension quantitative et qualitative considérable des missions de l'État ${ }^{85}$, notamment dans le domaine de la «prévoyance ${ }^{86}$ ». Selon Weber, la bureaucratie moderne doit son triomphe à sa supériorité technique sur des formes pré-modernes de l'administration, ou des formes d'exercice honorifique de celle-ci ${ }^{87}$. La " bureaucratisation universelle et inéluctable ${ }^{88}$ » ne concerne pas seulement des entités, comme les partis (de masse) ${ }^{89}$ et les administrations locales ${ }^{90}$, mais aussi des pays comme l'Amérique qui, jusqu'alors, ne connaissaient pas de corps de fonctionnaires formés et professionnels ${ }^{91}$. Dans ce contexte, Weber parle même d'une "européanisation de l'Amérique ${ }^{92}$ "; il estime que toute "démocratie d'un grand État», y compris donc la démocratie américaine, se développera pour devenir une " démocratie bureaucratisée »- de la même manière que le "socialisme rationnel"9 ", en tant qu' "alternative technique" au "capitalisme rationnel" ${ }^{4} "$, ne représente qu'un surcroît de bureaucratie.

III.

11 Le modèle de bureaucratie souvent cité de Weber, et pour la construction duquel il a utilisé des publications sur la bureaucratie prussienne ${ }^{95}$, présente les caractéristiques suivantes :

1. Les affaires administratives sont organisées «à la manière d'une entreprise ", elles sont exécutées de manière linéaire et selon des règles fixes. La direction administrative s'accomplit selon des règles abstraites («statutaires») qui rendent l'action administrative impersonnelle, mais plus calculable ;

2. les affaires administratives sont organisées selon le principe de la division du travail, selon des compétences fixes ${ }^{96}$ qui

3. sont intégrées dans une hiérarchie administrative ;

4. les moyens de l'administration et du fonctionnement ne sont pas la propriété personnelle des bureaucrates,

5. et le détenteur d'une fonction ne peut pas se l'approprier.

6. Le principe en vigueur est celui de l'archivage des dossiers [Aktenmäßigkeit] (règle de l'écrit).

7. Les membres d'une direction administrative ainsi caractérisée n'obéissent qu'aux devoirs de leur charge et sont soumis à une discipline administrative et à des contrôles rigoureux et 
homogènes. Ils sont recrutés sur la base d'une qualification professionnelle qui suppose une formation particulière et qui est attestée par des examens (diplômes) ${ }^{97}$. Le fonctionnaire spécialisé exerçant sa fonction comme profession principale est nommé, et employé par contrat ; il est tenu à une obligation de fidélité envers sa fonction, dont la signification est soulignée par des particularités analogues à des privilèges: un poste garanti à vie, un dédommagement prenant la forme d'un traitement, la perspective d'une carrière en fonction de l'ancienneté ainsi que la perspective d'une assurance vieillesse (pension) ${ }^{98}$.

Les caractéristiques énumérées ici composent une construction idéaltypique. Il s'agit donc d'une formation de pensée de nature heuristique. On néglige souvent cet aspect, peut-être aussi parce que les propos de Weber sur la bureaucratie moderne ont, dans la partie ancienne de la sociologie de la domination et dans ses textes politiques, plutôt un caractère descriptif, des «analyses historiques et structurelles " s'y mêlant aux "perspectives sociales globales et internes à l'organisation ${ }^{99}$ ". Si l'on accepte que l'idéaltype est au service d'un intérêt heuristique concret ${ }^{100}$, cette construction devient un instrument (de connaissance). Cela devient clair si l'on se rappelle que l'organisation rationnelle de l'administration et du travail sont pour Weber des structures centrales de la civilisation occidentale moderne. La rationalisation formelle et l'existence d'un type d'homme spécialisé ${ }^{101}$ renvoient à une objectivation de la domination des hommes sur les hommes, qui représente quant à elle la spécificité d'une «culture » centrée sur la «maîtrise du monde" et non sur "l'adaptation au monde ${ }^{102}$ " (perspective de comparaison historique et perspective sociale globale $)^{103}$. En ce sens, il existe un lien manifeste avec la théorie wébérienne de la rationalisation, à laquelle se rapportent, il est vrai, aussi bien sa sociologie de la domination que son modèle de bureaucratie. Si l'on se réfère à la problématique que Weber a formulée dans son "Avantpropos »:«Quel enchaînement de circonstances a conduit à ce que l'occident précisément, et lui seul, ait vu apparaître sur son sol des phénomènes culturels qui se sont inscrits dans une direction de développement qui a revêtu [...] une signification et une validité universelles ${ }^{104}$ ", et si l'on se rappelle que Weber compte au nombre de ces phénomènes culturels, entre autres, les fonctionnaires spécialisés, l'administration moderne (rationnelle) et l'État moderne ${ }^{105}$, il paraît évident que la problématique mentionnée plus haut ne peut être abordée sous l'angle du phénomène de civilisation qu'est la bureaucratie moderne que si l'on procède à une analyse comparative entre cette dernière et des formes pré-modernes de l'activité administrative ; pour ce faire, il faut des instruments appropriés qui permettent de démontrer la différence postulée du niveau de rationalité. Dans cette mesure, le modèle de la bureaucratie représente le type de bureaucratie rationnelle auquel on oppose, sous forme comparative (perspective structurelle), une forme pré-rationnelle (patrimoniale) de la bureaucratie ${ }^{106}$ ou des types alternatifs du point de vue structurel (comme l'administration par les notables), afin de pouvoir classer des formes historiquement existantes de l'activité rationnelle dans la catégorie du plutôt rationnel ou du plutôt non-rationnel ${ }^{107}$. Comme pour toute comparaison, on augmente le gain de connaissances, mais les propos ainsi obtenus ne peuvent pas être absolutisés, comme c'est assez souvent le cas dans l'utilisation de la citation qui suit. La "supériorité » de la bureaucratie moderne est en quelque sorte relativisée d'elle-même par la comparaison :

«Un mécanisme bureaucratique pleinement développé se comporte à leur égard [c'est-à-dire à l'égard des autres formes, N.d.l.A.] exactement comme une machine à l'égard des types non mécaniques de production de biens. Précision, rapidité, univocité, principe de l'archivage [Aktenkundigkeit], linéarité, discrétion, homogénéité, subordination rigoureuse, économies de frictions, de coûts objectifs 
et personnels sont portés à l'optimum dans le cas d'une administration rigoureusement bureaucratique, et spécifiquement : monocratique, exercée par des fonctionnaires individuels, par rapport à toutes les formes d'exercice collégial, honorifique ou comme activité secondaire ${ }^{108}$. »

13 Si le patrimonialisme politique fonctionne comme type contrastant au niveau de la perspective structurelle, il peut aussi servir de point de départ pour un mode d'observation historique pratiqué à la lumière de la théorie de la rationalisation et qui, de manière analogue à la problématique formulée par Weber dans son "Avant-propos", s'interroge sur l'enchaînement de circonstances grâce auquel l'État patrimonial a pu bénéficier, en Occident, d'opportunités pour devenir un État rationnel ${ }^{109}$. Sur la base de la partie ancienne de la sociologie de la domination, de celle des travaux consacrés par Weber à "l'éthique économique des religions universelles » et à l'aide de «séries de concepts idéaltypiques ${ }^{110}$ » de types fondamentaux traditionnels ou de types de formes particulières (ville, hiérocratie), on peut reconstituer des stades d'évolution idéaltypiques de la patrimonialisation en Occident, par contraste avec des stades d'évolution dans les empires moyen-orientaux ${ }^{111}$, afin de faire comprendre ainsi la vraisemblance de l'invraisemblable : la formation moderne de l'État ${ }^{112}$. Un double rôle semble échoir aux "séries de concepts idéaltypiques». D'une part, elles servent à comprendre le matériau historique à traiter ; d'autre part, on peut grâce à elles et à des fins heuristiques construire des "évolutions » sur le plan de l'idéaltype ${ }^{113}$. Il s'agit d'un procédé recevable, selon Weber, pour autant que l'on ne mélange pas «théorie et histoire ", que l'on ne les "confonde pas» et a fortiori que l'on ne combine pas «la construction idéale d'un développement et la classification conceptuelle d'idéaltypes de certaines structures culturelles [...] en une classification génétique » et que la « série des types qui en résulte » n'apparaisse pas comme une « succession historique de types obéissant à la nécessité d'une loi ${ }^{114}$ ».

On devrait à présent avoir clairement compris que le modèle de bureaucratie de Weber est construit en fonction de la problématique qui l'intéresse particulièrement au sein des sciences de la culture : celle de la rationalité spécifique à l'Occident ${ }^{115}$, qui est caractérisée par une série de phénomènes culturels propres à celui-ci. Ne pas en tenir compte conduit à des malentendus et des erreurs d'interprétation. Il arrive assez fréquemment que le modèle bureaucratique soit aussi présenté comme une description exacte de la "réalité ", parce que certains de ses caractères permettent de décrire les structures d'organisation de l'administration publique ou des principes traditionnels de la fonction publique professionnelle. C'est méconnaitre la fonction de l'idéaltype, comme le fait par ailleurs son application prescriptive dans l'esprit d'un modèle de la manière dont il faudrait modeler certaines organisations. Ce qui, en revanche, correspond à la fonction de l'idéaltype, c'est la procédure proposée pour le travail historique proprement dit, et que Weber lui-même a toujours respectée : "déterminer (dans chaque cas particulier) combien la réalité se rapproche ou s'écarte de ce tableau idéal ${ }^{116} »$. On pourrait citer comme exemple, en portant son regard vers les États-Unis d'une part, l'élection des fonctionnaires par les dominés ${ }^{117}$, d'autre part l'affirmation selon laquelle, en Prusse, la noblesse est parvenue « à affirmer sa position dominante et à désactiver les règles d'une carrière et d'une promotion au mérite ${ }^{118}$ ", ce qui conduit, dans les deux cas, à entreprendre des coupes dans le niveau de rationalité posé comme aune par l'idéaltype. D'une manière générale, il faut accepter l'idée que des phénomènes tels que la "bureaucratie coopérative », qui ne règne pas mais négocie, doivent être considérés comme un "passage à l'irrationnel ${ }^{119}$ " dans le cadre de 
référence fixé par Weber, ne serait-ce qu'en raison de la logique de construction qui caractérise le processus de rationalisation, et même si les négociations satisfont pour leur part aux critères de rationalité ${ }^{120}$.

IV.

15 Bien que Weber oriente sa typologie de la domination en fonction de certaines "différences de base dans les fondements généraux de la validité de la domination " (légitimité), il se concentre, dans ses plus anciennes études de sociologie de la domination, sur les piliers extérieurs de celle-ci, c'est-à-dire sur la question de l'organisation et $\mathrm{du}$ mode de fonctionnement de la domination ${ }^{121}$. Cela concerne en premier lieu le rapport entre le seigneur et la direction administrative, c'est-à-dire entre la direction politique et l'appareil bureaucratique, une thématique dans laquelle les dominés tiennent un rôle marginal. La place centrale est tenue par conséquent par le secteur de réalité constitué par les confrontations entre le seigneur et la direction ( (utte ») pour l'appropriation ou l'expropriation des pouvoirs de domination ou des moyens administratifs, ce qui, en raison de l'issue par principe incertaine de cette "lutte de pouvoir», permet de tenir compte de la catégorie de la contingence, hautement significative pour la compréhension que Weber a de l'évolution historique.

Lorsqu'il aborde la bureaucratie moderne, Weber discute du rapport tendu entre la politique (direction politique) et l'administration suivant trois thématiques principales. Le cas idéal de la (1) domination par le biais d'une direction administrative bureaucratique dans laquelle l'appareil a un caractère purement instrumental, peut, dans le quotidien administratif et sous certaines conditions, se muter en une (2) domination de la direction administrative, ou en une domination du corps des fonctionnaires dans laquelle l'appareil bureaucratique a pris son autonomie à l'égard de la direction politique. Enfin, il existe (3) une domination des fonctionnaires dans laquelle la direction politique n'est pas entre les mains de politiciens professionnels traditionnels ${ }^{122}$ montrant " passion, sentiment de responsabilité et sens de la mesure ${ }^{123}$ ", mais entre celles de fonctionnaires qui ne se préoccupent pas de politique ou de lutte pour le pouvoir ${ }^{124}$.

Parmi les conditions structurelles qui aident à assurer le primat de la politique, on trouve la séparation, constamment mise en relief par Weber, entre l'administration et les moyens objectifs de fonctionnement et d'administration. Pour ne pas se livrer entièrement à la supériorité des connaissances spécialisées et liées au service, le "dilettante» au pouvoir invente la solution de la formation d'une direction [Stabsbildung] ${ }^{125}$, c'est-à-dire du contrôle des experts par des experts (issus de l'organisation fonctionnelle), ce qui entraîne un conflit permanent entre spécialistes et généralistes. Il y a plus important encore que ce type de contrôle : les précautions et mesures institutionnelles à l'aide desquelles on peut réduire la supériorité dont dispose la bureaucratie sur le parlement en matière de connaissance, ou dont celui-ci reçoit des possibilités de contrôle efficaces sur l'administration ${ }^{126}$. Concrètement, Weber pense à l'institution de commissions parlementaires efficientes et à l'introduction du droit d'enquête ${ }^{127}$; d'une manière tout à fait générale, il juge nécessaire une progression de la parlementarisation et de la démocratisation (pour prévenir aussi une domination des fonctionnaires).

18 Même si, dans la bureaucratie moderne, il y a une séparation entre les fonctionnaires, d'un côté, les fonctions et les moyens d'administration de l'autre, même si les 
fonctionnaires sont recrutés selon des critères objectifs (qualification spécialisée), une bureaucratie revendiquant ses connaissances sur ses domaines de spécialisation, son service et ses procédures et se comportant ainsi en acteur politique, peut se dérober au contrôle exercé par l'exécutif et le parlement. Weber avait sous les yeux une évolution de ce type, avec l'exemple du constitutionnalisme fictif de la Prusse et de la Russie. Ce n'est pas seulement en raison de sa "spécialisation et [sa] formation technique rationnelle ${ }^{128}$ " que la bureaucratie a acquis un caractère « inéluctable » sans précédent, d'autant plus que ses "formes irrationnelles " (bureaucraties patrimoniales), une fois établies, comptaient au nombre des « entités sociales les plus difficile à détruire ${ }^{129}$ ». La question de l'influence qu'exerce une bureaucratie moderne professionnalisée et hautement spécialisée sur la politique a toujours été un thème de la recherche sur la bureaucratie ${ }^{130}$. Mais ce n'est que tout récemment que l'on a mené une tentative, aussi problématique soit-elle, pour étudier empiriquement le potentiel d'influence des bureaucraties ministérielles sur la politique (cabinet, direction de département; parlement) ${ }^{131}$. Mentionnons-la malgré tout brièvement ici, parce que l'on y opère une tentative instructive de distinguer trois possibilités de prise d'influence. Dans la "phase de décision pré-politique », la bureaucratie a la possibilité ou bien de mettre elle-même certains thèmes à l'ordre du jour (fixation d'agenda ou «bureaucratic free enterprise »), ou bien d'influencer selon ses propres conceptions la mission assignée par la politique (interaction stratégique); de la même manière, dans la "phase postpolitique » de la mise en œuvre, la possibilité de préciser les lois promulguées par des décrets d'application détaillés conformes à ses conceptions (drifting bureaucratique). Comme la recherche sur l'implémentation l'a suffisamment montré, l'administration exécutive a la possibilité d'interpréter ou de transformer à son gré la «volonté » du législateur ${ }^{132}$.

19 Weber a vécu de manière immédiate, sous la forme du Reich wilhelmien, la "domination des fonctionnaires ", c'est-à-dire le fait que les positions politiques dominantes soient occupées par des fonctionnaires. On pouvait l'expliquer, pour l'essentiel, par un parlementarisme insuffisamment développé qui ne se prêtait pas à servir de plateforme au choix des chefs politiques, ainsi que par l'existence d'une bourgeoisie qui ne détenait pas «la volonté de pouvoir» et ne produisait pas une culture politique adaptée qui aurait pu être utile à la "profession de la politique ${ }^{133}$ ». Weber, d'une manière générale, appelait de ses vœux une parlementarisation et en particulier un renforcement du parlement, ainsi qu'une abrogation de la clause d'incompatibilité de l'article 9 de la Constitution du Reich ${ }^{134}$. En effet, il discernait des facteurs décisifs qui expliquent l'importante proportion de fonctionnaires dans les positions politiques dirigeantes, et dont on peut aussi dire qu'ils ont été confirmés empiriquement. Comme l'a montré Lang pour la France et l'Allemagne (1871-2000), c'est-à-dire pour des pays dotés d'une tradition étatiste marquée, les membres du gouvernement se recrutaient plutôt dans l'administration lorsque la position du parlement était faible dans la structure de pouvoir de l'État ${ }^{135}$. Inversément, " avec le pouvoir du parlement et des partis (monta) aussi la part des hommes politiques exerçant des fonctions exécutives, qui provenaient de leur milieu ${ }^{136}$ ». Le " lien » étroit « entre la proportion élevée de fonctionnaires au sein du gouvernement et la clause d'incompatibilitée ${ }^{137}$ » est en outre confirmé par des études empiriques et comparatives internationales ${ }^{138}$ et vaut tout particulièrement pour le Reich wilhelmien ${ }^{139}$. En Allemagne fédérale, une fonction ministérielle est compatible avec un siège au parlement, c'est même pratiquement la règle, de la même manière que le chancelier 
fédéral est élu par le parlement. Dans cette mesure, le parlement a gagné une importance considérable pour ce qui concerne le recrutement de membres du gouvernement ${ }^{140}$, et à l'inverse «le passage de positions administratives d'élite vers la politique est extrêmement rare ${ }^{141} »$.

C'est contre la "domination des fonctionnaires » et la "politique bureaucratique " qu'étaient orientées les revendications wébériennes ${ }^{142}$ - introduction du suffrage universel égalitaire, parlementarisation - mais aussi sa proposition visant à faire élire les chefs politiques par plébiscite afin de faire contrepoids à une bureaucratie toute puissante dont il était convaincu du caractère inéluctable et de la capacité à survivre. Il se trouve cependant que c'est justement le concept wébérien de la domination charismatique des chefs que l'on n'a cessé de critiquer. Des interprètes éminents de Weber, comme Wolfgang J. Mommsen, ont même jadis affirmé qu'il avait contribué à donner « au peuple allemand la volonté mentale d'acclamer un chef, et donc aussi, dans cette mesure, Adolf Hitler ${ }^{143} »$. On a cependant pu lire depuis, chez Mommsen, que "derrière la construction unilatérale et à maints égards trop accaparante de la "démocratie plébiscitaire du chef" [plebiszitäre Führerdemokratie]» se trouve " également le motif du maintien possible d'un ordre libre dans un monde qui tend à la pétrification ${ }^{144} »$. Mommsen s'est ainsi rapproché de la position de Breuer, pour lequel le « concept de la démocratie plébiscitaire des chefs ne se situe pas dans une continuité avec le national-socialisme, mais représente au contraire la tentative - aussi problématique soit-elle - de domestiquer les tendances charismatiques de la démocratie de masse moderne ${ }^{145}$ ». A l'encontre de la sociologie wébérienne de la domination dans sa formulation tardive, on peut cependant argumenter avec Mommsen que même si elle se donne encore pour «neutre » dans de vastes domaines, elle présente cependant des tonalités politiques qui reflètent effectivement les expériences contemporaines (phase de naissance de la République de Weimar), mais qui sont également issues de la philosophie pessimiste de l'histoire de Weber. Celle-ci est perceptible dans l'idée que seule une figure de chef dotée de charisme pourrait retenir, voire empêcher le remplacement ou l'étouffement de la politique par des objectifs et des routines dirigés par la bureaucratie.

\section{V.}

21 Si l'on jette un regard sur la longue liste des textes consacrés au New Public Management (NPM), il semble que l'on ait assigné au modèle wébérien de bureaucratie le rôle de faire ressortir, comme un modèle contrastant, la supériorité et l'actualité du concept de $\mathrm{NPM}^{146}$. Ses caractéristiques sont pourtant aussi «étrangères à la réalité » que le modèle de bureaucratie idéaltypique de Weber, parce qu'elles restituent les intentions d'une philosophie de la réforme ${ }^{147}$. Si, en revanche, l'on fait appel à des études sur la recherche européenne, l'on a une opinion inverse sur la possibilité d'y intégrer Weber. Lepsius interprète ainsi les réflexions de Weber sur la bonne forme institutionnelle à donner à la relation entre parlement et gouvernement, entre bureaucratie et direction politique, entre efficience et démocratie à la fin de l'empire de Guillaume II, comme un programme d'analyse des institutions et de la politique des institutions ${ }^{148}$, qui peut aussi s'appliquer à la construction et à l'extension de l'Union Européenne. L'intérêt que Weber présente pour nous repose sur l'«actualité de ses problématiques » et sur la «base méthodologique de ses propositions pour une solution du problème ${ }^{149}$ ». Le 
programme de Weber pour une réorganisation de l'Allemagne sur la base d'une théorie des institutions contenait des propositions en vue d'un ordre institutionnel dans lequel, d'une part, dans la mise en œuvre de certaines propriétés du système une domination $\mathrm{du}$ parlement pouvait fixer des limites à une domination de la bureaucratie et où, d'autre part, en cas de conflits d'intérêt, on garantissait la capacité de prendre une décision. Mais si l'on garantissait la pluralité des intérêts et l'acceptation des conflits, une "poursuite consistante et à long terme de valeurs spécifiques ${ }^{150}$ " semblait toutefois possible ; on devrait dès lors procéder de manière analogue pour le modelage de l'Union Européenne et se demander « quelles compétences devaient être confiées à quel organe, par le biais de quelles possibilités ces compétences devaient être contrôlées et les nouveaux ordres européens légitimés, et à quelles conséquences vraisemblables ces ordres devraient s'attendre ${ }^{151}$. » Avec ce recours à Weber sur le plan de la théorie des institutions, mais aussi avec cette conception, qui se réfère elle aussi à lui, visant à considérer l'Union Européenne "sociologiquement comme un groupement de domination (européen et en devenir) ${ }^{152}$ ", Lepsius a montré que l'on peut analyser l'Union Européenne en utilisant Weber, même si son modèle de bureaucratie ne correspond pas à la forme de direction administrative existante, laquelle se distingue par des «directions composées de fonctionnaires multinationaux engagés dans une coopération internationale durable " et disposant en outre de structures de communication et de coordination informelles particulières ${ }^{153}$. L'actualité de Weber tient avant tout à la question qu'il pose : celle des conditions structurelles complexes qui tendent à encourager une prise d'autonomie de ces groupements de fonctionnaires", augmentant ainsi considérablement les chances d'une "politique bureaucratique » (dans le sens d'une domination du corps des fonctionnaires) dans le quotidien de l'administration. Pour simplifier, on peut mentionner les suivantes conditions structurelles générales d'une " politique bureaucratique » dans le cadre de l'Union Européenne. Le premier niveau des commissaires (collège des commissaires), avec leurs cabinets (dotés d'une fonction de direction), a en dessous de lui un deuxième niveau de l'appareil d'administration et des fonctionnaires (directions générales; services) que l'on peut décrire au mieux à l'aide de caractéristiques qui correspondent aux principes structurels traditionnels des organisations administratives bureaucratiques $^{154}$. A un troisième niveau s'est formé, avec le nombre incalculable des commissions, une "bureaucratie parallèle ${ }^{155}$ " constituée de réseaux administratifs et d'instances interétatiques gérées par des fonctionnaires («bureaucratie de fusion ${ }^{156}$ ») qui ne se plient pas aux structures bureaucratiques traditionnelles. Dans ces « réseaux horizontaux [...], les représentants du gouvernement » sont au contraire «intégrés, avec la participation de groupements privés et d'experts, dans un cadre de contact et de communication délimité sur le plan institutionnel, continuel et largement informel, qui dissout la structure étatique traditionnelle des espaces de compétence échelonnés hiérarchiquement et présentant une imputabilité relativement transparente de la responsabilité et de la compétence ${ }^{157}$ ». À ce troisième niveau, dans le passé comme aujourd'hui, est aussi accumulé le savoir spécialisé, de service et de procédure, c'est là que l'on « collecte et produit le savoir $»^{158}$, d'autant plus que par le biais des « réseaux d'administration interétatiques " (Wessels) qui y sont disponibles, on peut faire appel aux stocks d'information des acteurs nationaux ${ }^{159}$, de telle sorte que cette "supériorité [...] en matière de savoir » (Weber) permet une " politique bureaucratique » menée en bonne partie à l'écart du parlement : 
«Échappant à peu près à l'observation du public, à l'ombre de tous les contrôles politiques et démocratiques, traversés par une "philosophie" (ou une idéologie) managériale et technocratique "de résolution des problèmes", accessible aux pressions d'intérêts organisés, notamment néo-corporatistes, ce sont presque exclusivement des fonctionnaires et des "eurocrates", avec la participation d'experts scientifiques discrètement cooptés au sein de petits aréopages informels et opaques, qui prennent les décisions sur des questions sociales et politiques de grande portée [...] dans l'espace d'intégration européen ${ }^{160}$. »

\section{VI.}

Pour terminer cet aperçu consacré à "l'État et la bureaucratie » chez Weber, il faut rappeler avec Lepsius que la sociologie de Weber associe "l'activité sociale des acteurs à la structuration de la coordination de l'action et aux contextes du sens donné par ses orientations ${ }^{161} »$. Selon Lepsius, on ne doit cependant pas considérer cette tridimensionnalité comme une « analyse à plusieurs niveaux » :

"L'activité sociale se déroule au contraire "au niveau des acteurs" [...], dans des contextes d'actions structurés et se référant à des valeurs; le "niveau de la coordination de l'action" par des règles, des organisations, des groupements et des institutions se réfère toujours aux contextes qui apportent une légitimité au niveau du sens et à l'activité sociale d'acteurs ; le "niveau des valeurs culturelles" regroupe des combinaisons multiples d'idées de valeur dans une sélection extrêmement diverse, en tant qu'orientations de l'action et légitimations d'organisations ${ }^{162}$. »

Dans cette mesure, Weber, avec ses concepts, veut appréhender des processus et non des entités collectives, raison pour laquelle on lit aussi dans l'essai sur les catégories, en termes concis et ramassés : « Pour la sociologie, il n’y a, derrière la notion "d'État" [...], que le déroulement d'une activité humaine d'une espèce particulière ${ }^{163}$.» Weber a expliqué ce qu'il entend par là dans une lettre à Robert Liefmann le 9 mars 1920, en l'informant par ailleurs de sa volonté de mettre « un terme à l'activité ininterrompue qui travaille avec des concepts collectifs »:

« [...] la sociologie, elle aussi, ne peut se pratiquer qu'en partant de l'action d'un, de plusieurs ou d'un grand nombre d'individus, par conséquent de manière strictement “individualiste" quant à la méthode [...]. L'État n'est rien d'autre, au sens de la sociologie, que la chance que se produise une action spécifique sous des modes déterminés, une action de la part de personnes individuelles déterminées. Ce n'est rien d'autre [...]. L'élément "subjectif' dans cette affaire consiste en ce que cette action s'oriente d'après des représentations déterminées. L'élément "objectif", en ce que nous - les observateurs - nous jugeons que la chance existe que cette action, telle quelle s'oriente, d'après ces représentations, s'effectuera., Si cette chance n'existe plus, "l'État" n'existe plus ${ }^{164}$."

Un tel concept processuel de l'État, centré sur des orientations d'action déterminées ${ }^{165}$, est taillé à la mesure de transformations à la fois conscientes et induites par des "changements de signification ${ }^{166}$ ", de telle sorte que même des transformations radicales qui dénotent une "modification du rôle de l'État ${ }^{167}$ " peuvent ainsi être appréhendées par son intermédiaire. La cristallisation presque exclusive de l'intérêt sur le modèle de bureaucratie wébérien a pratiquement empêché que l'on prête attention à sa conception de l'État, centrée sur l'action. 


\section{BIBLIOGRAPHIE}

Albrow,M. (1972) :Bürokratie, Munich.

Anter,A. (1996) :Max Webers Theorie des modernen Staates. Herkunft, Struktur und Bedeutung, $2^{\mathrm{e}}$ éd., Berlin.Un chapitre de ce livre est publié en traduction française dans ce numéro $7 \mid 2010$ de Trivium : « L'histoire de l'Etat comme histoire de la bureaucratie » [NDLR].

Anter,A. (2001) :«

Von der politischen Gemeinschaft zum Anstaltsstaat. Das Monopol der legitimen Gewaltsamkeit » , dans : Hanke, E. / Mommsen, W. J. (éd.) :Max Webers Herrschaftssoziologie, Tübingen, p. 121-138.

Anter,A. (2004) :Die Macht der Ordnung. Aspekte einer Grundkategorie des Politischen, Tübingen.

Anter, A. / Breuer, S. (éd.) (2007) :Max Webers Staatssoziologie, Baden-Baden.

Bach,M. (1995) :«

Ist die europäische Einigung irreversibel ? Integrationspolitik als Institutionenbildung in der Europäischen Union

», dans : Nedelmann, B. (éd.) :PoIitische Institutionen im Wandel,Opladen, p. 368-391.

Bach,M. (1999) :

Die Bürokratisierung Europas. Verwaltungseliten, Experten und politische Legitimation in Europa, Francfort-sur-le-Main / NewYork.

Bach,M. (2005) :«

Europa als bürokratische Herrschaft. Verwaltungsstrukturen und bürokratische Politik in der Europäischen Union

", dans : Schuppert, G. F. / Pernice, I. / Haltern, U. (éd.) :Europawissenschaft

, Baden-Baden, p. 575-607.

Balthasar,H. U. von (1961) :Die großen Ordensregeln, Zurich / Cologne.

Barberis,P. (2005) :« Whitehall Mandarins and the British Elite Network 1870-1945 »,

Jahrbuch für Europäische Verwaltungsgeschichte, 17, p. 1-26.

Beetham,D. (1987) :Bureaucracy, Milton Keynes.

Benz,A. (1995) : Der Beitrag der Spieltheorie zur Analyse des kooperativen Verwaltungshandelns ", dans : Dose, N. / Voigt, R. (éd.) :Kooperatives Recht, Baden-Baden, p. 297-328.

Benz,A. (2001) :Der moderne Staat. Grundlagen der politologischen Analyse, Munich / Vienne.

Bleek,W. (1972) :

Von der Kameralausbildung zum Juristenprivileg. Studium, Prüfung und Ausbildung der höheren Beamten des allgemeinen Verwaltungsdienstes in Deutschland im 18. und 19. Jahrhundert , Berlin.

Bogumil, J. / Jann,W. (2005) :

Verwaltung und Verwaltungswissenschaft in Deutschland. Einführung in die Verwaltungswissenschaft , Wiesbaden.

Bohne,E. (1981) :

Der informale Rechtsstaat. Eine empirische und rechtliche Untersuchung zum Gesetzesvollzug unter besonderer Berücksichtigung des lmmissionsschutzes , Berlin. 
Boldt,H. (2001) :« "Den Staat ergänzen, ersetzen oder sich mit ihm versöhnen?" Aspekte der Selbstverwaltungsdiskussion im 19. Jahrhundert » , dans : Hanke, E. / Mommsen, W. J. (éd.) :Max Webers Herrschaftssoziologie, Tübingen, p. 139-165. Breuer,S. (1990) :« Rationale Herrschaft. Zu einer Kategorie Max Webers », Politische Vierteljahresschrift, 31, p. 4-32 ;trad. fr. : « La domination rationnelle. A propos d'une catégorie de Max Weber », dans : Trivium 7 | 2010 [NDLR].

Breuer,S.(1991) : Max Webers Herrschaftssoziologie, Francfort-sur-le-Main / New York.

Breuer,S.(1993) : « Max Webers Staatssoziologie », Kölner Zeitschrift für Sozio

logie und Sozialpsychologie, 45, p. 199-219.

Breuer,S.(1994) : « Das Charisma des Führers », dans : id. : Bürokratie und Charisma. Zur Politischen Soziologie Max Weber, Darmstadt, p. 144-175.

Breuer,S.(1994a) : «Die vier reinen Typen der Demokratie. Ein Vorschlag zur Systematisierung » , dans : id. :Bürokratie und Charisma. Zur Politischen Soziologie Max Webers, Darmstadt, p. 176-187.

Breuer,S.(1998) : Der Staat. Entstehung, Typen, Organisationsstadien, Reinbek.

Breuer,S.(2000) : «Das Legitimitätskonzept Max Webers », dans : Willoweit, D. / Müller-Luckner, E. (éd.) : Die Begründung des Rechts als historisches Problem, Munich, p. 1-17.

Breuer,S.(2003) : « Max Webers Parteisoziologie und das Problem des Faschismus », dans : Albert, G. / Bienfait, A. / Sigmund, S. / Wendt, C. (éd.) : Das Weber

-Paradigma. Studien zur Weiterentwicklung von Max Webers Forschungsprogramm , Tübingen, p. 352-370.

Breuer,S.(2004) : «Von der sozialen Staatslehre zur Staatssoziologie », dans : Anter, A. (éd.) : Die normative Kraft des Faktischen. Das Staatsverständnis Georg Jellineks, Baden-Baden, p. 89-112.

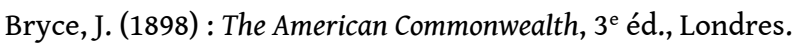

Budäus, D. (1998): « Von der bürokratischen Steuerung zum New Public Manage ment - Einführung », dans :Budäus, D. / Conrad, P. / Schreyögg, G. (éd.) :New Public Management , Berlin / New York, p. 1-9.

Bücker, A. / Schlacke, S. (2000) : «Die Entstehung einer “ politischen Verwaltung” durch EG-Ausschüsse - Rechtstatsachen und Rechtsentwicklungen » , dans : Joerge, C. / Falke, J. (éd.) :

Das Ausschusswesen der Europäischen Union. Praxis der Risikoregulierung im Binnenmarkt und ihre rechtliche Verfassung , Baden-Baden, p. 161-256.

Cullity, J. P. (1967) : « The Growth of Governmental Employment in Germany, 1882-1950 », Zeitschrift für die gesamte Staatswissenschaft, 123, p. 201-217.

Delley, J.-D. / Derivaz, R. /Mader, L. / Morand, Ch.-A. / Schneider,D. (1984) : Grundstückerwerb durch Ausländer in der Schweiz. Empirische Untersuchungen der Lex Furgler , Bern / Stuttgart.

Derlien, H.-U. (1989) : « Die selektive Interpretation der Weberschen Bürokratie theorie in der Organisations- und Verwaltungslehre »,Verwaltungsarchiv, 22, p. 319-329.

Derlien, H.-U. (1991) : « Die Staatsaffinität der Exekutivpolitiker der Bundesrepu blik. Zur Bedeutung der Bürokratie als Sozialisationsfeld » , dans : Hartwich, H.-H. / Wewer, G. (éd.) : 
Regieren in der Bundesrepublik II. Formale und informale Komponenten des Regierens in den Bereichen Führung, Entscheidung, Personal und Organisation , Opladen, p. 169-178.

Derlien, H.-U. (1994) : « Bürokratie in der Literatur und Soziologie der Moderne. Über Kafka und Max Weber », dans : Anz, T. / Stark, M. (éd.) : Die Modernität des Expressionismus, Stuttgart / Weimar, p. 44-61.

Derlien, H.-U. (2002) : « Öffentlicher Dienst im Wandel », dans : König, K. (éd.) : Deutsche Verwaltung an der Wende zum 21. Jahrhundert, Baden-Baden, p. 229-253.

Derlien, H.-U. (2003) : « Mandarins or Managers? The Bureaucratic Elite in Bonn, 1970 to 1987 and Beyond ", Governance, 16, p. 401-428.

Derlien, H.-U. (2003a) : « German Public Administration : Weberian Despite "Modernization" », dans : Tummala, K. K. (éd.) : Comparative Bureaucratic Systems, Lanham / Boulder / New York / Oxford, p. 97-122.

Derlien, H.-U. / Frank, S. (2004) : « Öffentlicher Dienst und Gewerkschaftssystem im Wandel », Die Verwaltung, 37, p. 295-326.

Derlien, H.-U. / Lang, F. (2005) : « Die Verwaltungseliten in der Bundesrepu blik Deutschland und der V. Französischen Republik », Jahrbuch für Europäische Verwaltungsgeschichte, 17, p. 109-147.

Dogan, M. (1989) : «Introduction : Selecting Cabinet Ministers », dans : id. (éd.) : Pathways to Power : Selecting Rulers in Pluralist Democracies, Londres, p. 1-18.

Ellwein, Th. (1987) : « Entwicklungstendenzen der deutschen Verwaltung im 19. Jahrhundert », Jahrbuch zur Staats- und Verwaltungswissenschaft, 1, p. 13-54.

Ellwein, Th. (1990) : «Die Fiktion der Staatsperson - eine Skizze », dans : Ellwein, Th. / Hesse, J. J. (éd.) : Staatswissenschaften: vergessene Disziplin oder neue Herausforderung ?, Baden-Baden, p. 99-110.

Ellwein, Th. (1993) : Der Staat als Zufall und als Notwendigkeit. Die jüngere Ver waltungsentwicklung in Deutschland am Beispiel Ostwestfalen-Lippe, vol. 1 : Die Öffentliche Verwaltung in der Monarchie 1815-1918, Opladen.

Ellwein, Th. (1994) : Das Dilemma der Verwaltung. Verwaltungsstruktur und Ver waltungsreformen in Deutschland, Mannheim / Leipzig / Wien / Zurich.

Ellwein, Th. / Hesse, J. J. (1997) : Der überforderte Staat, Francfort-sur-le-Main.

Falke, J. (2000) : « Komitologie-Entwicklung, Rechtsgrundlagen und erste empirische Annäherung », dans : Joerges, C. / Falke, J. (éd.) : Das Ausschusswesen der Eu ropäischen Union. Praxis der Risikoregulierung im Binnenmarkt und ihre rechtliche Verfassung , Baden-Baden, p. 43-159.

Flora, P. / Alber, J. / Eichenberg, R. / Kohl, J. / Kraus, F. / Pfenning, W. / Seebohm, K. (1983) : State, Economy and Society in Western Europe 1815-1975. A Data Handbook in two Volumes, vol. 1 : The Growth of Mass Democracies and Welfare States, Francfort-sur-le-Main / Londres / Chicago.

Forsthoff, E. (1959) : Rechtsfragen der leistenden Verwaltung, Stuttgart; et plus particulièrement « Einleitung », p. 9-21, « Die Daseinsvorsorge als Aufgabe der modernen Verwaltung » , p. 22-34 (extrait deDie Verwaltung als Leistungsträger, 1938).

Gephart, W. (1990) : «Juristische Ursprünge in der Begriffswelt Max Webers », Rechtshistorisches Journal, 9, p. 343-362. 
Gerhardt, V. (1996) : Vom Willen zur Macht. Anthropologie und Metaphysik der Macht am exemplarischen Fall Nietzsches, Berlin / New York.

Grottrup, H. (1976) : Die kommunale Leistungsverwaltung, Stuttgart / Berlin / Cologne / Mayence.

Hanke, E. (2001) : « Max Webers “ Herrschaftssoziologie”. Eine werkgeschichtliche Studie », dans : Hanke, E. / Mommsen, W. J. (éd.) : Max Webers Herrschaftssoziologie, Tübingen, p. 19-46.

Hanke, E. (2005) : « Einleitung », dans : MWG I/22-4, p. 1-91.

Hattenhauer, H. (1980) : Geschichte des Beamtentums, Cologne / Berlin / Bonn / Munich.

Heffter, H. (1950) : Die deutsche Selbstverwaltung im 19. Jahrhundert. Geschichte der Ideen und Institutionen, Stuttgart.

Hermes, S. (2003) : Soziales Handeln und Struktur der Herrschaft. Max Webers verstehende historische Soziologie am Beispiel des Patrimonialismus, Berlin.

Hermes, S. (2004) : « Das Recht einer “ soziologischen Rechtslehre”. Zum Rechtsbegriff in Max Weber Soziologie des Rechts ", Rechtstheorie, 35, p. 195-231.

Hermes, S. (2006) : Der « Staat » als Institution. Max Webers soziologische Begriffsbildung im Kontext der Rechts- und Staatswissenschaften, dans : Lichtblau, K. (éd.) : Max Webers « Grundbegriffe ». Kategorien der kultur- und sozialwissenschaftlichen Forschung, Wiesbaden, 2006, p. 185-216.

Hess, H. (1993) : Mafia. Ursprung, Macht und Mythos, Fribourg / Bâle / Vienne.

Hesse, J. J. (1987): « Aufgaben einer Staatslehre heute », Jahrbuch zur Staats- und Verwaltungswissenschaft, 1, p. 55-87.

Hill, H. (1997): « Neue Organisationsformen in der Staats- und Kommunalverwaltung », dans : Schmidt-Assmann, E. / Hoffmann-Riem, W. (éd.) : Verwaltungsorganisationsrecht als Steuerungsressource, Baden-Baden, p. 65-101.

Hintze, O. (1926) : « Max Webers Soziologie » (recension de : Grundrisse der Sozialökonomik. III. Abt.: Wirtschaft und Gesellschaft), Schmollers Jahrbuch, 50, p. 83-95; trad. fr. : « La sociologie de Max Weber ", dans : Hintze (1991), p. 35-50.

Hintze, O. (1981 [1911]) : « Der Beamtenstand », dans : Krüger, K. (éd.) : Otto Hintze. Beamtentum und Bürokratie, Göttingen, p. 16-77 (première publication : Vorträge der Gehe-Stiftung zu Dresden, vol. 3, Dresde, 1911).

Hintze, O. (1981 [1910]) : « Der Commissarius und seine Bedeutung in der allgemeinen Verwaltungsgeschichte ", dans : Krüger, K. (éd.) : Otto Hintze. Beamtentum und Bürokratie, Göttingen, p. 78-112 (première publication : Historische Aufsätze Karl Zeumer zum 60. Geburtstag dargebracht, Weimar, 1910, p. 493-528).

Hintze, O. (1991) : Féodalité, capitalisme et Etat moderne. Essais d'histoire sociale comparée, choisis et présentés par Hinnerk Bruhns, Paris : Editions de la Maison des sciences de l'homme.

Hoffmann, F. (1981) : « Organisation-Umwelt-Beziehungen in der Organisationsforschung. Klassische und neoklassische Organisationstheorien ", dans : Kieser, A. (éd.) :

Organisationstheoretische Ansätze, Munich, p. 103-111.

Hood, C. C. (1976) : The Limit of Administration, Londres.

Jaspers, K. (1949 [1931]) : Die geistige Situation der Zeit, Berlin, repr. de la 5 éd. revue en été 1932. En français : La situation spirituelle de notre époque, trad. de l'allemand par J. Ladrière et W. Biemel, Paris : Desclée de Brouwer, 1951. 
Jessen, R. (1992) : « Unternehmerschaft und staatliches Gewaltmonopol. Hüttenpolizei und Zechenwehren im Ruhrgebiet (1870-1914) », dans : Lüdtke, A. (éd.) : «Sicherheit » und "Wohlfahrt ». Polizei, Gesellschaft und Herrschaft im 19 und 20. Jahrhundert, Francfort-sur-le-Main, p. 161-186.

Jessen, R. (1995) : « Private und öffentliche Formen sozialer Kontrolle in der Geschichte der Industrialisierung », dans : Sack, F. / Voss, M. / Frehsee, D. / Funk, A. / Reinke, H. (éd.) : Privatisierung staatlicher Kontrolle: Befunde, Konzepte, Tendenzen, Baden-Baden, p. 139-155.

Kafka, F. (1975 [1919]) : In der Strafkolonie. Eine Geschichte aus dem Jahre 1914, Berlin. En français : « À la colonie pénitentiaire », dans : David, Cl. (éd.) : Un artiste de la faim et autres récits, Paris : Gallimard, 1990

Kieser, A. (1999) : « Max Webers Analyse der Bürokratie », dans : id. (éd.) : Organisationstheorien, $3^{\mathrm{e}}$ éd., Stuttgart, p. 39-64.

Knöbl, W. (1998) : Polizei und Herrschaft im Modernisierungsprozeß. Staatsbildung und innere Sicherheit in Preußen, England und Amerika 1700-1914, Francfort-sur-le-Main / New York.

König, K. (1999) : Verwaltungsstaat im Übergang. Transformation, Entwicklung, Modernisierung, BadenBaden.

Kocka, J. (1981) : « Otto Hintze, Max Weber und das Problem der Bürokratie », Historische Zeitschrift, 233, p. 65-105 ; trad. fr. : «Otto Hintze, Max Weber et le problème de la bureaucratie », dans : Trivium 7 | 2010 [NDLR]

Koselleck, R. (1975) : Preußen zwischen Reform und Revolution. Allgemeines Landrecht, Verwaltung und soziale Bewegung von 1791-1848, $2^{\mathrm{e}}$ éd., Stuttgart.

Kuhlmann, S. (2004) : « Evaluation lokaler Verwaltungspolitik : Umsetzung und Wirksamkeit des Neuen Steuerungsmodells in den deutschen Kommunen », Politische Vierteljahresschrift, 45, p. 370-394.

Lang, F. (2005) : Die Verwaltungselite in Deutschland und Frankreich 1871-2000. Regimewechsel und Pfadabhängigkeiten, Baden-Baden.

Lange-Kirchheim, A. (1986) : « Alfred Weber und Franz Kafka », dans : Demm, E. (éd.) : Alfred Weber als Politiker und Gelehrter, Stuttgart, p. 113-149.

Lee, K.-H. / Raadschelders, J. C. N. (2005) : « Between Amateur Government and Career Civil Service : The American Administrative Elite in Cross-Time and Cross-National Perspectives », Jahrbuch für Europäische Verwaltungsgeschichte, 17, p. 201-222.

Lepsius, M. R. (1995) : « Max Weber und das Programm einer Institutionenpolitik », Berliner Journal für Soziologie, 5, p. 327-333.

Lepsius, M. R. (1995a) : « lnstitutionenanalyse und Institutionenpolitik», dans : Nedelmann, B. (éd.) : Politische Institutionen im Wandel, Opladen, p. 392-403.

Lepsius, M. R. (2000) : « Die Europäische Union als rechtlich konstituierte Verhal tensstrukturierung ", dans : Dreier, H. (éd.) :

Rechtssoziologie am Ende des 20. Jahrhunderts. Gedächtnissymposion für Edgar Michael Wenz , Tübingen, p. 289-305.

Lepsius, M. R. (2003) : «Eigenart und Potenzial des Weber-Paradigmas », dans : Al bert, G. / Bienfait, A. / Sigmund, S. / Wendt, C. (éd.) :

Das Weber-Paradigma. Studien zur Weiterentwicklung von Max Webers Forschungsprogramm , Tübingen, p. 32-41. 
Lichtblau, K. (2000): “ "Vergemeinschaftung” und "Vergesellschaftung” bei Max Weber. Eine Rekonstruktion seines Sprachgebrauchs », Zeitschrift für Soziologie, 29, p. 423-443.

Lübbe, W. (1991) : Legitimität kraft Legalität. Sinnverstehen und Institutionenanalyse bei Max Weber und seinen Kritikern, Tübingen.

Lüdtke, A. (1992) : " "Sicherheit” und "Wohlfahrt". Aspekte der Polizeigeschichte », dans : id. (éd.) : «Sicherheit » und « Wohlfahrt ». Polizei, Gesellschaft und Herrschaft im 19. und 20. Jahrhundert, Francfort-sur-le-Main, p. 7-33.

Mayer, O. (1908) : Die juristische Person und ihre Verwertbarkeit im öffentlichen Recht, Staatsrechtliche Abhandlungen. Festgabe für Paul Laband zum fünfzigstenJahrestage der Doktor-Promotion , Tübingen, p. 1-4.

Mayntz, R. (1968) : « Max Webers Idealtypus der Bürokratie und die Organisationssoziologie » , dans : id.(éd.) :Bürokratische Organisation, Cologne / Berlin, p. 27-35 ;trad. fr. : « L’idéaltype wébérien de la bureaucratie et la sociologie des organisations » dans : Trivium 7 | 2010 [NDLR].

Mayntz, R. (1995) : « Gesellschaftliche Modernisierung und die veränderte Rolle des Staates », dans : Max-Planck-Gesellschaft. Jahrbuch, p. 57-70.

Mayntz, R. (1997) : Soziologie der öffentlichen Verwaltung, $4^{\mathrm{e}}$ éd., Heidelberg.

Michels, R. (1911) : Zur Soziologie des Parteiwesens in der modernen Demokratie. Untersuchungen über die oligarchischen Tendenzen des Gruppenlebens, Leipzig.

Mommsen, W. J. (1965): « Diskussion über "Max Weber und die Machtpolitik" », dans : Stammer, o. (éd.) : Max Weber und die Soziologie heute, Tübingen, p. 130-138.

Mommsen, W. J. (1974) : Max Weber und die deutsche Politik 1890-1920, $2^{\mathrm{e}}$ éd., Tübingen. Trad. fr. : Max Weber et la politique allemande, 1890-1920, trad. de l'allemand par J. Amsler, Paris : PUF, 1985.

Mommsen, W. J. (2001) : « Politik im Vorfeld der “Hörigkeit der Zukunft”. Politi sche Aspekte der Herrschaftssoziologie Max Webers", dans : Hanke, E. / Mommsen, W. J. (éd.) : Max Webers Herrschaftssoziologie, Tübingen, p. 303-319.

Morsey, R. (1957) : Die Oberste Reichsverwaltung unter Bismarck 1867-1890, Münster.

Nelson, M. (1982) : « A Short, Ironic History of American National Bureaucracy », The Journal of Politics, 44, p. 747-778.

Pollit, C. / Bouckaert, G. (2004) : Public Management Reform. A Comparative Analysis, $2^{\mathrm{e}}$ éd., Oxford. Popitz, H. (1992) : Phänomene der Macht, $2^{\mathrm{e}}$ éd., Tübingen.

Portinaro, P. P. (2001) : « Amerika als Schule der politischen Entzauberung. Eliten und Parteien bei Max Weber », dans : Hanke, E. / Mommsen, W. J.(éd.) : Max Webers Herrschaftssoziologie, Tübingen, p. 285-302.

Pressman, J. L. / Wildavsky, A. (1973) : Implementation, Berkeley / Los Angeles / Londres.

Puntscher Riekmann, S. (1998) : Die kommissarische Neuordnung Europas. Das Dispositiv der Integration, Vienne / New York.

Puntscher Riekmann, S. (2000) : « Die Meister und ihr Instrument. Institutionenkon flikte und Legitimationsprobleme in der Europäischen Union », dans : Bach, M. (éd.) : Die Europäisierung nationaler Gesellschaften, p. 130-151. 
Raadschelders, J. C. N. / Rutgers, M. R. (1996) : « The Evolution of Civil Service Systems », dans : Bekke, H. A. G. M. / Perryt, J. L. / Toonen, Th. A. J. (éd.) : Civil Service Systems in Comparative Perspective, Bloomington / Indianapolis, p. 67-99.

Recktenwald, H. C. (1977) : « Umfang und Struktur der öffentlichen Ausgaben in säkularer Entwicklung », Handbuch der Finanzwissenschaft, vol. 1, Tübingen, p. 713-752.

Reichard, C. (1995) : « Von Max Weber zum “ New Public Management” - Ver waltungsmanagement im 20. Jahrhundert»

, dans : Halblützel, P. / Haldemann, T. / Schedler, K. / Schwaar, K. (éd.) :

Umbruch in Politik und Verwaltung. Ansichten und Erfahrungen zum New Public Management in der Schweiz , Bern / Stuttgart /Vienne, p. 57-79.

Reichard, C. (2002) : «Verwaltung als öffentliches Management », dans : König, K. (éd.) : Deutsche Verwaltung an der Wende zum 21. Jahrhundert, Baden-Baden, p. 255-277.

Rose, R. (1975): « On Priorities of Government : A Developmental Analysis of Public Policies », European Journal of Political Research, 4, p. 247-289.

Roth, G. (1987) : « Direkte Demokratie und Parteienverfall in den Vereinigten Staaten », dans : id. : Politische Herrschaft und persönliche Freiheit. Heidelberger Max Weber-Vorlesungen 1983, Francfortsur-le-Main, p. 31-57.

Roth, G. (2005) : « Europäisierung, Amerikanisierung und Yankeetum. Zum New Yorker Besuch von Max und Marianne Weber 1904 », dans : Schluchter, W. / Graf, F. W. (éd.) : Asketischer Protestantismus und der « Geist » des modernen Kapitalismus, Tübingen, p. 9-31.

Samier, E. (2001) : « Demandarinisation in the New Public Management : Examining Changing Administrative Authority from a Weberian Perspective ", dans : Hanke, E. / Mommsen, W. J. (éd.) : Max Webers Herrschaftssoziologie, Tübingen, p. 235-263.

Schluchter, W. (1972) : Aspekte bürokratischer Herrschaft. Studien zur Interpre tation der fortschreitenden Industriegesellschaft, Munich.

Schluchter, W. (1979) : Die Entwicklung des okzidentalen Rationalismus. Eine Analyse von Max Webers Gesellschaftsgeschichte, Tübingen.

Schluchter, W. (1980) : « Bürokratie und Demokratie. Zum Verhältnis von politischer Effizienz und politischer Freiheit bei Max Weber ", dans : id. : Rationalismus der Weltbeherrschung. Studien zu Max Weber, Francfort-sur-le-Main, p. 75-133.

Schluchter, W. (1980a) : « Der autoritär verfasste Kapitalismus. Max Webers Kritik am Kaiserreich », dans : id. : Rationalismus der Weltbeherrschung. Studien zu Max Weber, Francfort-surle-Main, p. 134-169.

Schmid, G. / Treiber, H. (1975) : Bürokratie und Politik. Zur Struktur und Funktion der Ministerialbürokratie in der Bundesrepublik Deutschland, Munich.

Schmoller, G. (1894 sq.): Acta Borussica. Denkmäler der Preußischen Staatsverwaltung im 18. Jahrhundert, Königliche Akademie der Wissenschaften (éd.), Reihe A : Die Behördenorganisation und die allgemeine Staatsverwaltung Preußens im 18. Jahrhundert, vol. 1 sqq., Berlin.

Schnapp, K.-U. (2004) : Ministerialbürokratien in westlichen Demokratien. Eine vergleichende Analyse, Opladen.

Schönberger, C. (1997) : Das Parlament im Anstaltsstaat. Zur Theorie parlamentarischer Repräsentation in der Staatslehre des Kaiserreichs (1871-1918), Francfort-sur-le-Main. 
Schuppert, G. F. (2000) : Verwaltungswissenschaft. Verwaltung, Verwaltungsrecht, Verwaltungslehre, Baden-Baden.

Silberman, B. S. (1993) : Cages of Reason. The Rise of the Rational State in France, Japan, the United States, and Great Britain, Chicago / Londres.

Speer, H. (1978) : Herrschaft und Legitimität. Zeitgebundene Aspekte in Max Webers Herrschaftssoziologie, Berlin.

Spittler, G. (1980) : «Abstraktes Wissen als Herrschaftsbasis. Zur Entstehungsgeschichte bürokratischer Herrschaft im Bauernstaat Preußen », Kölner Zeitschrift für Soziologie und Sozialpsychologie, 32, p. 574-604.

Steinberg, R. (1978) : «Faktoren bürokratischer Macht », Die Verwaltung, 3, p. 309-334.

Stollberg-Rilinger, B. (1986) : Der Staat als Maschine. Zur politischen Metaphorik des absoluten Fürstenstaates, Berlin.

Stolleis, M. (1992) : Geschichte des öffentlichen Rechts in Deutschland, vol. 2 : Staatsrechtslehre und Verwaltungswissenschaft 1800-1914, Munich.

Süle, T. (1988) : Preußische Bürokratietradition. Zur Entwicklung von Verwaltung und Beamtenschaft in Deutschland 1871-1918, Göttingen.

Toiler, A. E. (1999) : « Die Implementation europäischer Politik durch Ausschüsse : Zur Funktionsweise und politikwissenschaftlichen Relevanz der Komitologie », Österreichische Zeitschrift für Politikwissenschaft, 28, p. 333-352.

Treib, O. (2004) : Die Bedeutung der nationalen Parteipolitik für die Umsetzung europäischer Sozialrichtlinien, Francfort-sur-le-Main.

Treiber, H. (1998) : « lm Schatten des Neukantianismus : Norm und Geltung bei Max Weber », dans : Brand, J. / Strempel, D. (éd.) : Soziologie des Rechts. Festschrift für Erhard Blankenburg zum 60. Geburtstag, Baden-Baden, p. 245-254.

Trotha, T. von, (1995) : « Ordnungsformen der Gewalt oder Aussichten auf das Ende des staatlichen Gewaltmonopols », dans : Nedelmann, B. (éd.) : Politische Institutionen im Wandel, Opladen, p. 129-166.

Trotha, T. von (1995a) : « Staatliches Gewaltmonopol und Privatisierung. Notizen über gesamtstaatliche Ordnungsformen der Gewalt », dans : Sack, F. / Voss, M. / Frehsee, D. / Funk, A. / Reinke, H. (éd.) : Privatisierung staatlicher Kontrolle: Befunde, Konzepte, Tendenzen, Baden-Baden, p. 14-37.

Trotha, T. von (1997) : « Zur Soziologie der Gewalt », dans : id. : Soziologie der Gewalt, p. 9-56.

Trotha, T. von (2000) : « Was ist Recht? Von der gewalttätigen Selbsthilfe zur staatlichen Rechtsordnung ", Zeitschrift für Rechtssoziologie, 21, p. 327-354.

Tyrell, H. (1980) : « Gewalt, Zwang und die Institutionalisierung von Herrschaft : Versuch einer Neuinterpretation von Max Webers Herrschaftsbegriff », dans : Pohlmann, R. (éd.) : Person und Institution. Helmut Schelsky gewidmet, Würzburg, p. 59-92

Tyrell, H. (1981) : « Ist der Weber'sche Bürokratietypus ein objektiver Richtigkeitstypus? Anmerkungen zu einer These von Renate Mayntz », Zeitschrift für Soziologie, 10, p. 38-49.

Tyrell, H. (1999) : « Physische Gewalt, gewaltsamer Konflikt und “ der Staat” Überlegungen zu neuerer Literatur », Berliner Journal für Soziologie, 9, p. 269-288. 
Weber, A. (1982 [1910]) : « Der Beamte », dans : id. : Haben wir Deutschen nach 1945 versagt? Politische Schriften, éd. par C. Dericum, Francfort-sur-le-Main, p. 29-52.

Wessels, W. (2000) : Die Öffnung des Staates. Modelle und Wirklichkeit grenz überschreitender Verwaltungspraxis 1960-1995, Opladen.

Willke, H. (1987) : « Entzauberung des Staates. Grundlinien einer systemtheoreti schen Argumentation "Jahrbuch zur Staats- und Verwaltungswissenschaft, 1, p. 285-308.

Windelband, W. (1921[1878]) : « Über Friedrich Hölderlin und sein Geschick (Nach einem Vortrage in der akademischen Gesellschaft zu Freiburg i. B. am 29. November 1878) », dans : id. : Präludien. Aufsätze und Reden zur Philosophie und ihrer Geschichte, $7^{\mathrm{e}}$ et $8^{\mathrm{e}}$ éd., Tübingen, p. 230-259.

Wunder, B. (1978) : Privilegierung und Disziplinierung. Die Entstehung des Berufs beamtentums in Bayern und Württemberg (1780-1825), Munich / Vienne.

Wunder, B. (1986) : Zur Geschichte der Bürokratie in Deutschland, Francfort-sur-le-Main.

Wunder, B. (1993) : « Das Prüfungsprinzip und die Entstehung der Beamtenschaft in Deutschland ", Jahrbuch für Wirtschaftsgeschichte, 1, p. 11-26.

Wunder, B. (2005) : « Prüfungsgrundsatz und Adelsprivilegien : das Scheitern eines Elitenwandels in der deutschen Verwaltung 1806-1914 ", Jahrbuch für Europäische Verwaltungsgeschichte , 17, p. 51-83.

Abréviations utilisées pour les œuvres de Max Weber citées en langue allemande :

GPS : Weber, M. (1971) : Gesammelte Politische Schriften, éd. par J. Winckelmann, $3^{\mathrm{e}}$ éd., Tübingen.

GARS : Weber, M. (1972) : Gesammelte Aufsätze zur Religionssoziologie, $6^{e}$ éd., Tübingen, vol. 1.

GASS : Weber, M. (1988) : Gesammelte Aufsätze zur Soziologie und Sozialpolitik, éd. par Marianne Weber, $2^{\mathrm{e}}$ éd., Tübingen.

MWG I/8 : Weber, M. (1998) : Wirtschaft, Staat und Sozia1politik. Schriften und Reden 1900-1912 , éd. par W. Schluchter, Tübingen. « [Die wirtschaftlichen Unternehmungen der Gemeinden]. Diskussi onsbeitrag auf der Generalversammlung des Vereins zur Sozialpo1itik am 28. September 1909 » , dansid., p. 360-366.

MWG I/10: Weber, M. (1989) : Zur Russischen Revolution von 1905. Schriften und Reden 1905-1912, éd. par W. J. Mommsen en coll. avec D. Dahlmann, Tübingen.

MWG I/15: Weber, M. (1984) : Zur Politik im Weltkrieg. Schriften und Reden 1914-1918, éd. par W. J. Mommsen en coll. avec $\mathrm{G}$

\section{NOTES}

1. Weber, WuG, p. 29 [E\&S, p. 97]. Dans les commentaires de cette définition, Weber dit explicitement que ce concept de l'État vaut pour l'État moderne entièrement développé (ibid., p. 30). Weber lui-même indique (ibid., p.636; MWG I/22-4, p. 411) que le concept moderne de l'État n'est pas applicable à des formes pré-modernes de structures politiques (par exemple: féodalisme, "État des ordres [Ständestaat] »), même s'il utilise de manière très libre le concept d' «État ». Voir Breuer (1993), p. 207. - Parmi les autres textes illustrant son concept de l'État, citons MWG I/17 («Politik als Beruf»), notamment p. 157-160, p. 162 sqq., p. 166 sq. : « [...] que l'État moderne est un groupement de domination de type institutionnel (anstaltsmäßig) qui s'est 
efforcé et a réussi à monopoliser, à l'intérieur d'un territoire, la violence physique légitime comme moyen de domination, et qui a rassemblé à cette fin entre les mains de ses dirigeants les moyens matériels de l'entreprise, tandis qu'il expropriait tous les fonctionnaires qui, en vertu des droits propres des «états » [Stände] disposaient jadis de façon autonome de ces moyens, et qu'il s'est mis à leur place au sommet de l'État.» [trad. de C.Colliot-Thélène, SP, p. 127 sq.]. Également : MWG I/15 (« Parlament und Regierung im neugeordneten Deutschland »), p. 450-454, 460-467 [trad. fr. : OP, p. 307 sq.] ; MWG I/16 (« Deutschlands künftige Staatsform »). Par ailleurs : WG, p. 289 sqq., GARS I, p. 3 sq. (« Vorbemerkung ») : « D’une façon générale, l'État au sens d'une institution [Anstalt] politique dotée d'une "constitution" établie rationnellement, d'un droit fixé rationnellement et d'une administration conduite par des agents spécialisés conformément à des règles établies et rationnelles, c'est-à-dire des "lois" ». [SR, p. 492 sq.]. Cf. ibid. ("Einleitung »), p. 267 sq., 272 sq. ; MWG I/19, p. 119 sq., 124 sq. ; GARS I («Zwischenbetrachtung »), p. 547 sq. [trad. fr. : SR, p. 410-460]; MWG I/19, p. 491 sq.

2. Anter (1996), p. 13.

3. WL, p. 466 sq. [ETS, p. 325 sq.].

4. WuG, p. 429 sqq.

5. WuG, p. 28 [E\&S, p. 27].

6. Sur les difficultés qui résultent d'une application universelle du concept de groupement politique par le principe territorial intégré à la définition, voir Hermes (2003), p. 90. Sur la signification du principe territorial à l'égard du concept de politique, voir Tyrell (1999), p. 283.

7. WuG, p. 28 [E\&S, p. 27]; WL, p. 468 sq. [ETS, p. 330]. Il faut rappeler, avec Hermes (2004), p. 222 sq., que chez Weber, l'ordre représente un concept générique « dont le droit est considéré comme une sous-espèce ». Voir WL, p. 445, note 1 [ETS, p. 354]. Les « institutions statutaires » de l'État (rationnel) sont, de ce point de vue, du droit. Sur la problématique associée au concept d'ordre : Anter (2004), p. 86 sqq.

8. WuG, p. 449 : «Depuis la révélation charismatique de nouveaux commandements, le chemin le plus direct de l'évolution mène, via l'imperium, à la création de droit par le "statut" convenu et imposé ».

9. WL, p. 440 [ETS, p. 347] ainsi que Gephart (1990).

10. WL, p. 440 [ETS, p. 347] ; WuG, p. 6 sq. [E\&S, p. 39 sq.].

11. WL, p. 466 [ETS, p. 386].

12. Mayntz (1968) ; Derlien (1989). Ce que l'on appelle la « thèse de l'efficience » est répandu, voir par exemple Beetham ([1987], p. 14 sqq.) qui écrit à ce propos : «Weber believed that the defining characteristics of bureaucracy were also necessary conditions for administrative or organizational efficiency ». En règle générale, on présente aussi le modèle de la bureaucratie comme un prototype de «schéma de la fin et des moyens ». Voir Hoffmann (1981). Kieser (1999) est une exception, entre autres parce qu'il présente parfois les unes à côté des autres, sans les lier, des interprétations fort différentes émises par des experts de Weber.

13. Hermes (2005) ; en se référant à Schönberger (1997).

14. Hermes (2005), p. 18-21 ; Schönberger (1997), p. 21-120, ainsi que déjà Speer (1978), p. 37-41. Également Mayer (1908), p. 53 sq., 68-70. Pour les juristes dont il est question dans le texte, le «pouvoir de volonté de l'État» est toujours un pouvoir de domination. La «conception monocentrique, focalisée sur le pouvoir d'État comme centre de volonté dominatrice concrétisait l'État [...] dans l'activité organique de mise en ordre et d'administration menée par les organes de l'État incarnant le pouvoir d'État » (Hermès [2005], p. 19 ; ainsi que Hanke [2005], p. 4 sq.) Voir aussi Stolleis (1992), p. 330 sq. («Méthode juridique» et théorie constitutionnelle du Reich); également Ellwein (1990). L'approche de Laband suscita des oppositions. Otto von Gierke et Fritz Stoerk, notamment, critiquèrent sa manière de « réduire l'État au point de focalisation juridique d'un pouvoir d'État exclusivement exercé du haut vers le bas» (Stolleis [1992], p. 345 ; également Hanke [2005], p. 9 sq. ; Boldt [2001]). Sur l'influence de Jellinek sur Weber, cf. Breuer (2004). Il 
faut toutefois rappeler que Weber, pour ce qui concerne son concept du pouvoir et de la domination (WuG, p. 28, p. 544 ; MWG I/22-4, p. 135) est également lié à des classiques que les ombres du Reich impérial n'atteignaient pas. Lorsque Gerhardt ([1996], p. 18) attire l'attention sur le fait que le pouvoir n'est « compréhensible que dans des contextes intentionnels » et dit à ce propos que « de la même manière qu'aucun pouvoir ne peut être représenté sans une volonté agissant à travers lui, la volonté se décompose elle aussi lorsqu'elle perd son pouvoir ; elle devient une volonté "impuissante" et donc un simple souhait ». Cette cristallisation conceptuelle sur la volonté, qui caractérise chez Weber le concept de pouvoir tout comme celui de domination (comme expression de la volonté et par là-même comme "commandement"), montre justement à quel point il fait appel aux classiques. Pour Kant aussi, au bout du compte, la «volonté » est « un commandement » (cité d'après Gerhardt (1996), p. 222 sq.). L'affinité élective avec Saint Augustin est étonnante (Gerhardt [1996], p. 44) : «Le pouvoir véritable, on peut comprendre Augustin ainsi, est un pouvoir de domination (dominandi potestas). Il part d'une volonté et est orienté sur une autre volonté, ne misant sur rien d'autre, en l'occurrence, que sur l'intelligence du soumis ». Étonnant aussi, le parallélisme entre la définition de la domination chez Weber (WuG, p. 544 ; MWG I/22-4, p. 135) et l'idéal d'obéissance des bénédictins : «L'acte est accompli en fonction de l'obéissance, lorsque la chose commandée est exécutée ; selon la volonté, lorsque celui qui obéit désire la même chose que celui qui commande; selon l'intelligence, lorsqu'il ressent la même chose que celui-ci, en sorte qu'il considère l'ordonné comme tout à fait bon. Et l'obéissance est imparfaite si, à côté de l'exécution, n'existe pas aussi l'uniformité du désir et de la sensation entre celui qui commande et celui qui obéit » (Balthasar [1961], p. 376). Rappelons que Weber, lui aussi, (WuG, p. 544 sq., MWG 1/22-4, p. 135 sq.) tient déjà compte pour la « chaîne causale entre l'ordre et son exécution", de "l'empathie », autant que de "l'intuition », ou même de la "persuasion rationnelle». Dans cette mesure, la définition de la domination (WuG, p.544; MWG I/22-4, p. 135) comporte déjà une sorte "d'accord de légitimité ». Sur le concept de domination chez Weber, cf. aussi Tyrell (1980).

15. Cette réduction du champ de vision conditionnée par la terminologie («État institutionnalisé ») pourrait également être mise en relief dans la critique de Weber par Knöbl ([1988], p. 353 sq.) dans le contexte des analyses comparatives des sociétés, celles de la Prusse, de l'Angleterre et de l'Amérique. Avec l'Angleterre et l'Amérique, il a consciemment intégré dans la comparaison des pays qui n'ont pas produit de bureaucratie patrimoniale, même si les premiers germes en ont existé en Angleterre (WuG, p. 390, [SD, p. 192], 560 ; MWG I/22-4, p. 180).Voir aussi Hermès (2003), p. 185 sq., 191.

16. WuG, p. 6 [E\&S, p. 42, trad. mod.].

17. Hesse (1987), p. 79 ; cf. aussi Anter (1996), p. 95 ; Wilke (1997) ; Hill (1997), p. 65 sq.

18. Schluchter (1972), p. 74.

19. WuG, p. 128 [E\&S, p. 297].

20. Dans les commentaires au $\S 15$ des Concepts fondamentaux de la sociologie, (WuG, p. 28, alinéa 1 [E\&S p. 94]) Weber parle par exemple de la « continuité d'une activité en finalité »).

21. MWG I/15, p. 452 sq.; MWG I/17, p. 163 sq. [OP, p. 323 sq.].

22. Particulièrement instructifs sur ce point, les propos que l'on trouve chez Hermes (2003), notamment p. 84-92, 140-151, qui - contrairement à Breuer (1993) -, ne part pas de deux notions de l'État conçues différemment. La divergence résulte du fait qu'Hermes refuse une lecture qui déduit de l'essai sur les catégories une opposition entre institution et groupement et considère plutôt l'institution comme le «cas spécifique rationnel du groupement» (Hermes [2003], p. 84-91). On peut d'autre part opposer à Hermes l'idée que chez Weber, il existe aussi, à côté du concept étroit, moderne, de l'institution, un autre concept d'institution tel qu'il était donné en Chine avec le «charisme de l'institution». Cf. Breuer, « Wege zum Staat», dans : Anter /Breuer (2007), p. 57-77.

23. WL, p. 468 sqq. [ETS, p. 389 sq.] ; WL, p. 469 [ETS, p. 391]. 
24. WuG, p. 27 [E\&S, p. 91].

25. WL, p. 466 [ETS, p. 387].

26. WuG, p. 30 [E\&S, p. 99].

27. Lichtblau (2000), p. 433, qui rattache les distinctions conceptuelles exposées non seulement à une théorie sociale de la rationalisation, mais aussi à une théorie sociale de la différenciation (WL, p. 461 [ETS, p. 378 sq.]).

28. Voir en particulier les explications sur ce point : «En raison de sa direction administrative, un groupement est toujours, à un quelconque degré, un groupement de domination » (WuG, p. 28 [E\&S, p. 96]).

29. WuG, p. 26, § 12, sous l'alinéa 2 [E\&S, p. 89].

30. Sur le concept de légitimité chez Weber, voir Breuer (2000). Spécialement sur la « légitimité fondée sur la légalité », Lübbe (1991), et sur la «légitimité et la légalité dans la sociologie compréhensive ", Hermes (2003), p. 92 sqq.

31. Au débat mené à l'heure actuelle, qui met en perspective la « fin du monopole de la violence de l'État» (par exemple Trotha [1995] et [1999]), on peut opposer l'idée que ce monopole était aussi tout à fait fragile au XIX ${ }^{e}$ siècle. Des études historiques indiquent non seulement que l'État bureaucratique institutionnalisé (y compris et justement en Prusse) a connu jusqu'à la Première Guerre mondiale, pour ce qui concerne l'exercice du contrôle social par la police considérée comme une «institution clef du monopole de la violence dont disposait l'État » (Jessen [1985], p. 147), des formes d'émergence pré-modernes, mais aussi que l'effectif policier réel était modeste, avec un clivage caractéristique entre la ville et la campagne, et que son augmentation n'a lieu qu'au cours de la deuxième moitié du XIX siècle (Ellwein [1993], p. 475 sqq.; Lüdtke [1992]). Ellwein ([1993], p. 196 sqq., 405 sqq.) parle ainsi d'une «situation policière globale à plusieurs strates» et souligne particulièrement que la "police du système pénitentiaire [Vollzugspolizei] n'est en aucun cas dominée par l'État, mieux, que ses missions elles-mêmes n'ont pas été définies par l'État ", mais qu'une " coexistence d'intérêt étatique et communal pour la police » a été déterminante. Même si l'on est disposé à reconnaître les succès considérables obtenus par l'État en matière de monopolisation, ces succès ont manifestement des frontières immanentes: «Les formes non-étatiques de contrôle social ont été conservées sous forme de reliques de situations anciennes, sont apparues de nouveau dans les conditions de la société industrialisée urbaine, et se sont associées de manière spécifique aux instances de contrôle de l'État » (Jessen [1995], p. 150.) Parmi ces reliques, on trouve par exemple la domination foncière à l'est de l'Elbe (Anter [1996], p. 79 sqq. ; Schluchter [1980], p. 159 sqq.) ou le duel, et au nombre des formes nouvelles, à l'époque, les « milices de protection des puits » dans le secteur minier (Jessen [1992]). Le «caractère de compromis entre la domination étatique et la domination patrimoniale » en Prusse, qui s'est exprimé de manière exemplaire dans la domination foncière de l'est de l'Elbe et dans la figure du chef de district [Landrat], ne doit pas nécessairement être interprété comme une faiblesse de l'État. L'État prussien s'est servi de la «domination intermédiaire ", représentée par le chef de district, sur le territoires et les gens, mais a aussi su s'attacher étroitement ce dernier pour bureaucratiser ensuite cette «instance, qui était traditionnellement la moins bureaucratique, peut-être, de toute l'administration » (Süle, [1988], p. 40 sq. ; ainsi que Heffter [1950], p. 554 sq., également Koselleck [1975], p. 680 sqq. : « Part de la noblesse et des bourgeois dans l'administration provinciale et dans les postes de chef de district "; Spittler [1980]). D’une manière générale, il faudrait rappeler dans ce contexte le jugement de Weber : "Qu'un "État" n' "existe" que lorsque et là où les moyens de coercition de la communauté politique sont réellement plus puissants que ceux de toute autre communauté, c'est une notion étrangère à la sociologie. » (WuG, p. 184 [E\&S, t. 2, p. 18]).

32. WuG, p. 514 ; MWG I/22-1, p. 204. Sur ce concept correspondant, sur le fond, au « groupement politique », cf. Hermes (2003), p. 90. 
33. MWG I/17, p. 159 sq., 166 sq. [E\&S, p. 356 sq., 369 sq]. Weber refuse explicitement l'idée de définir l'État ou le groupement politique en fonction d'une une finalité de l'activité de groupement en mutation constante; il voit plutôt dans le moyen de l'exercice de la force (WuG, p. 30 [E\&S, p. 98 sq.]) l'élément unique et persistant des groupements politiques. Il s'est ainsi ouvert la possibilité d'une perspective d'histoire universelle, ce qui n'aurait pas été possible avec un concept de l'État centré sur l'Europe (Hanke [2001], p. 27). Cela dit, Otto Hintze ([1926], p. 86 [trad. fr. : (1991), p. 35-50]) a déjà souligné le fait que la sociologie de la domination chez Weber peut être, en son "noyau, qualifié de "sociologie de l'État" ». Weber lui-même a qualifié sa sociologie de la domination de "théorie de l'État et de la domination » (MWG II/8, p. 450). Sur Weber et Hintze, avec un regard sur la «bureaucratie », cf. Kocka (1981). Même si Weber refuse de parler de finalités de l'État, il a tout de même relevé des «fonctions de base de l'État » (WuG, p. 516) qui coïncident avec celles que Rose (1975) a identifiées dans son étude d'histoire comparée comme des missions centrales typiques de l'État. Pour l'Allemagne, on peut également discerner les missions centrales typiques de l'État à l'aide des tableaux présentés par Lange ([2005], p. 31) sur les « ministères allemands de 1871 à 2002 par année de fondation ».

34. MWG I/17, p. 160 [« La profession et la vocation de savant »].

35. Hermes (2003), p. 149 sq.

36. WuG, p. 670 sq., p. 453 ; MWG I/22-4, p. 514 sq.

37. Hess (1993), p. 189-213, notamment p. 200 sqq.

38. Popitz (1992), p. 63 ; Tyrell (1999).

39. WuG, p. 30, commentaires, alinéa 3 [E\&S, p. 99].

40. WuG, p. 370. Cf. aussi Trotha (2000), p. 349 : «Toutes les formes et institutions visant à régler les conflits se trouvent à l'ombre de la prétention au monopole de la force par l'État, et avec lui à l'ombre du droit de l'État. "

41. WuG, p. 17 [E\&S, p. 66].

42. WuG, p. 545 ; MWG I/22-4, p. 138 sq.

43. Hermes (2003), p. 147.

44. WuG, p. 544 ; MWG I/22-4, p. 135.

45. Hermes (2004), p. 229.

46. WL, p. 331 sqq.

47. WuG, p. 181 sqq. [E\&S, p. 397 sq].

48. Il s'avère que le concept du droit de Weber est tout sauf conçu ou défini de manière homogène. Le droit est par exemple d'une part un " ordre garanti par la contrainte ", de l'autre une représentation, existant dans l'esprit des gens, du caractère obligatoire d'un ordre (validité du droit), avec la possibilité, donnée du même coup, que les gens s'orientent aussi sur cet ordre, ce qui augmente aussi, pour finir, la probabilité qu'il soit respecté. Voir Hermes (2004), p. 218 sqq. Sur le concept de validité, cf. Treiber (1998).

49. WuG, p. 16 [E\&S, p. 65 sq.]. Treiber (1998), p. 246 sq., avec des références à la fonction de modèle de Rickert et Windelband pour la conception qu'a Weber du concept de validité.

50. Hermes (2004), p. 229. Sur le lien conceptuel de la sociologie de la domination et de la sociologie du droit, voir Hanke (2005), p. 79 sqq., ainsi que Schluchter (1979), p. 122 sqq.

51. WuG, p. 549 ; MWG I/22-4, p. 146 ; également Hanke (2005), p. 71 sqq.

52. WL, p. 476.

53. Sur la domination légale, rationnelle, voir Breuer (1991), p. 192 sq.

54. Trotha (1997), p. 12, note 4.

55. Mayntz (1968), p. 30, identifie le type de bureaucratie de Weber comme un «type objectif de justesse » [objektiver Richtigkeitstypus] (WL, p. 433 sq. [ETS, p. 336 sq.]), formulant les conditions qui doivent être réunies pour l'exercice durable et techniquement efficace de la domination légale.

56. Tyrell (1981), p. 40. 
57. Le principe d'obéissance comprend aussi le cas où le fonctionnaire doit agir même contre sa propre conviction, pour autant que l'ordre donné par un supérieur disposant $d u$ pouvoir d'instruction est conforme au droit (légal).

58. WuG, p. 507, 565. Également Breuer (1990), p. 17.

59. MWG $1 / 17$, p. 183 sqq. («La profession et la vocation de savant », avec une typologie des «spécialistes de l'administration», parmi lesquels on compte aussi les «juristes» (ibid., p. 185 sq.) Entre autres: Bleek (1972); Hattenhauer (1980); Süle (1988), Wunder (1978). Rappelons, dans ce contexte, une citation de Weber sur l'importance des juristes: "Et la naissance de "l'État" occidental moderne, comme des "Églises" occidentales a été pour une part tout à fait essentielle l'œuvre de juristes. » (GARS I, p. 272 [SR, p. 374] ; MWG I/19, p. 124).

60. WuG, p. 563 ; MWG I/22-4, p. 187.

61. GPS, p. 273 [trad. fr. : « Droit de vote et démocratie en Allemagne », OP, p. 251 sq.] ; MWG I/15, p. 377 ; sur le formalisme, cf. aussi WuG, p. 130 [E\&S, p. 301].

62. Tyrell (1981), p. 46.

63. Sur cette métaphore, voir Stollberg-Rilinger (1986) ainsi que Anter (1996), p. 210 sqq.

64. Breuer (1991), p. 210 sqq.

65. WuG, p. 681 ; MWG I/22-4, p. 542.

66. WuG, p. 682 ; MWG I/22-4, p. 545. [Le terme « suivre » rend ici l'allemand « mitlaufen » N.d.T.]

67. WuG, p. 682 ; MWG I/22-4, p. 545.

68. WuG, p. 130 [E\&S, p. 301].

69. Mayntz (1997), p. 60 sq. ; également Steinberg (1978).

70. Tyrell (1981), p. 46 sq.

71. Breuer (1990), p. 20, avec des références à Albrow (1972), p. 62 sqq. et Schluchter (1972), p. 122 sqq. Voir aussi Derlien (1989) et Mayntz (1968).

72. Breuer (1990), p. 20.

73. Derlien (1994), p. 51 sq.

74. König (1999), p. 49 sqq., notamment p. 53 sqq.

75. Breuer (1990), p. 20, avec d'autres indications bibliographiques.

76. Alfred Weber (1982 [1910]), p. 30. Comme l'a montré Lange-Kirchheim (1986), Kafka a utilisé l'essai d'Alfred Weber publié en 1910 « Der Beamte » («Le fonctionnaire ») comme modèle de son texte Dans la colonie pénitentiaire, publié pour la première fois en 1919. Voir Derlien (1994), p. 59 sq.

77. MWG I/15, p. 464 [« Domination des fonctionnaires et leadership politique », OP, p. 336], ainsi que MWG I/10, p. 269 sqq.

78. GASS, p. 414 ; MWG I/8, p. 363.

79. MWG, I/15, p. 464 [OP, p. 336].

80. WuG, p. 556 sqq. ; MWG I-22/4, p. 169 sqq.

81. WuG, p. 562 sq. ; MWG I/22-4, p. 187 sqq.

82. WuG, p. 560 ; MWG I/22-4, p. 180.

83. WuG, p. 559 ; MWG I/22-4, p. 176 sq.

84. Entre autres, Cullity (1967) ; Flora (1983), p. 11-26 (Makro-Modell of Europe de Rokkan), p. 16-18 (State Formation), p.194, p. 213-218 (personnel 1871-1975), p. 383-393 (dépenses de l'État 1872-1975) ; Recktenwald (1977) ; Schmid / Treiber (1975), p. 83 sqq. ; Wunder (1986). De manière concise et expressive, Wunder (1993), p. 16 : «La croissance sans cesse constatée du corps des fonctionnaires au XIX ${ }^{\mathrm{e}}$ siècle était certes partiellement due à de nouvelles embauches, mais découlait avant tout de la titularisation d'employés de l'État jusqu'alors embauchés à titre seulement privé et de l'intégration à la fonction publique d'organisations existantes mais non étatiques, comme celles du secteur de l'éducation, des employés communaux et d'autres groupes analogues. Les plus grands taux de croissance concernaient, au XIX ${ }^{\mathrm{e}}$ siècle, l'école, la poste et les chemins de fer, dont aucun n'avait été nationalisé au début du siècle et dont seul les chemins de 
fer constituaient une activité économique nouvelle d'un point de vue technique ». Voir aussi Ellwein (1987). Raadschelders / Rugters (1996) dessinent un schéma en cinq phases de l'évolution du personnel de la bureaucratie (centrale) («service public»), à propos des pays d'Europe de l'Ouest. Ils utilisent ici comme "critère » «l'idéaltype » wébérien de la bureaucratie moderne ; compte tenu des données empiriques disponibles, ils se cantonnent à «such characteristics as formal rules and procedures, use of written documents, hierarchical structure of offices, recruitment and appointment, pre- and postentry training, career-system, expertise, salary and pension facilities, and legal protection of the position. » (Raadschelders / Rugters [1996], p. 71).

85. WuG, p. $559 \mathrm{sqq}$; MWG I/22-4, p. 177 sqq., 182 sqq. Sur la dimension quantitative du changement de structure et de fonction de l'activité de l'État, Schmid / Treiber (1975), p. 74 sqq : les données contenues dans les tableaux 1 et 2 (dépenses de l'État du point de vue séculier 1890-1962) confirment en gros la tendance constatée par Adolf Wagner de l'augmentation absolue et relative des dépenses de l'État. C'est justement dans la période la plus récente (motsclef : « dégraisser l'État ", "État rentable ») que l'on mène des tentatives pour réduire l'ampleur des missions et celle du personnel. Voir sur ce point, par exemple, Benz (2001), p. 192-202 ; König (1999), p. 16-34; Derlien (2002). Derlien / Frank ([2004], p. 296-306) esquissent des «cycles d'évolution des effectifs du service public »; leurs données permettent aussi de déterminer l'ampleur de la baisse des effectifs dans le service public. Il faudrait aussi tenir compte du personnel des autorités de régulation. - On trouve un aperçu des diverses propositions de réformes entre autres chez Bogumil / Jann (2005), p. 183 sq. ; Ellwein (1994); Schuppert (2000), p. 917 sqq. On trouve des réflexions courantes sur la "transformation de la "qualité de l'État" " par exemple chez Benz (2001), p. 223 sqq. ; Ellwein / Hesse (1997), p. 161 sqq.

86. Chez Jaspers (1949[1931]), p. 58 sq., tourné sous l'angle philosophique, pour être ensuite repris par Forsthoff (1959/1938).

87. WuG, p. 561 sq; MWG I/22-4, p. 185 sq.

88. MWG I/15, p. 607 [« Le socialisme », OP, p. 465] ; GASS, p. 413 ; MWG I/8, p. 361.

89. WuG, p. 559 sq. ; MWG I/22-4, p. 177 ; MWG I/15, p. 451 [OP, p. 308] ; par ailleurs Bryce (1898) ; Michels (1911) ; Portinaro (2001). Sur la bureaucratisation attendue des partis en Amérique, Weber s'est manifestement trompé, dès lors que les partis de patronage n'y étaient "pas "strictement bureaucratiques" » (WuG, p. 669) mais patrimoniaux (Roth [1987], p. 34).

90. GASS, p. 412 sqq. ; MWG I/8, p. 360 sqq. Pour la période située entre la fin du XIX et le début du XXe siècles : Gröttrup (1976), p. 13 sqq. (« La croissance des dépenses publiques, et notamment communales »); également Wunder (1986), p. 83 sqq. (bureaucratisation de l'administration municipale, activités multipliées dans le domaine des prestations de service, vers 1880-1914); p. 127 sqq. (évolution quantitative dans l'entre-deux-guerres, y compris dans les collectivités territoriales).

91. MWG I/15, p. 603 [OP, p. 464 sq.]. Sur l'évolution de la fonction publique en Amérique : Lee / Raadschelders (2005) ; également Nelson (1982).

92. Roth ([2005], p. $12 \mathrm{sq}$.) donne un rapide commentaire sur ce que Weber entend par l'« européanisation » de l'Amérique.

93. WuG, p. 129 [E\&S, p. 300].

94. Schluchter (1972), p. 90 ; MWG I/15, p. 606 sq. [OP, p. 469].

95. WuG, p. 124 sq. [E\&S, p. 288 sq., voir en particulier p. 290]. Voir par exemple Schmoller (1894 sq.) ; Hintze (1981[1911]) ; Hanke (2005), p. 30 sq. Une raison de cette dominance de la Prusse pourrait avoir tenu au fait qu'en Allemagne, « l'administration de l'État [...] au XIX ${ }^{\mathrm{e}}$ siècle, même sous l'empire, était presque exclusivement une administration régionale. Le Reich luimême n'eut après sa fondation en 1871 [...] qu'une administration rudimentaire. De toutes les administrations régionales, l'administration prussienne était la plus nombreuse [...]. Raison pour laquelle le modèle prussien était déterminant. » Voir Wunder (2005), p. 55. Également Morsey, 1957. 
96. La division du travail mène à la spécialisation et ainsi à la disparition de "l'homme de civilisation » (WuG, p. 578), ce que déplorait déjà Windelband (1921[1878]) dans sa «conférence sur Hölderlin». On peut aussi citer à ce propos l'allusion au traité de Samier (2001), qui se réclame de Weber et dans lequel les réformes accomplies en Angleterre sous la doctrine du New Public Management (NPM) ont été interprétées comme un processus de « dé-mandarisation ». Ce processus a transformé les anciens serviteurs de l'État du senior civil service, qui respectaient une éthique professionnelle spécifique et pour lesquels on utilise la notion wébérienne de "mandarins", en managers efficaces désormais exposés à une politisation accrue et à des contrôles de grande ampleur, auxquels ils ont réagi par des "succès " à court terme et mesurables en termes économiques. Sur la formation du " gentleman of the service », cf. Silberman (1993), p. 363 sq.

97. Sur le système des examens, voir Wunder (1993) et (2005).

98. WuG, p. 125 sq. [E\&S, p. 291 sq.]. La séparation en deux pans du modèle de bureaucratie, une face d'organisation et une face liée au personnel, permet de renvoyer à la distinction introduite par Silberman ([1993], p. IX sqq., p. 4 sqq.) entre une « rationalisation liée à l'organisation et [une rationalisation] orientée sur la profession ", que représentent, au sein du type État [Staatlichkeit] rationnel, deux chemins d'évolution différents à l'aide desquelles on peut par exemple caractériser les évolutions en Prusse et en France, d'une part, en Angleterre et aux États-Unis de l'autre. Voir aussi sur ce point Breuer (1998), p. 166 sq., 175 sqq.

99. Schluchter (1972), p. 78 ; Schluchter (1980), p. 90 sq.

100. WL, p. 195 [ETS p. 186 sq.].

101. Le prototype en est le juriste ayant suivi une formation rationnelle et systématique dans les universités.

102. Comme jadis en Chine. Cf. Schluchter (1980), p. 100.

103. Schluchter (1980), p. 90 sq.

104. GARS I, p. 1. [SR, p. 489].

105. GARS I, p. 3 sq. [SR, p. 492].

106. Schluchter (1980), p. 99 ; Hermes (2003), p. 131 sqq., notamment p. 135 sqq. Voir aussi l'allusion de Weber (WuG, p. 124 [E\&S, p. 290]) : «C'est intentionnellement que nous partons ici de la forme spécifiquement moderne de l'administration afin de la confronter par la suite aux autres. » De cette manière, Weber se "protège » aussi des modèles par paliers, construits dans l'esprit de « l'école historique ».

107. Hanke (2001), p. 34 sqq.

108. WuG, p. 561 sq. ; MWG I/22-4, p. 185.

109. Hermes (2003), p. 135.

110. Hermes (2003), p. 136.

111. Hermes (2003), p. 231, avec référence à Breuer (1998), p. 274 sqq. ; ainsi que Breuer (1991), p. 191 sqq.

112. Hermes (2003), p. 138.

113. En détail chez Hermes (2003), p. 131 sqq.

114. WL, p. 204 [ETS p. 199].

115. Dans le seul « Avant-propos » de quinze «bonnes » pages (GARS I), on trouve quarante-six occurrences des mots « rationnel " et "rationalité »; « rationalisation" apparaît neuf fois, « rationalisme » en revanche trois fois.

116. WL, p. 191 [ETS, p. 181].

117. WuG, p. 554 ; MWG I/22-4, p. 163 sqq. ; p. 138, note 26.

118. Wunder (2005), p. 76 ; Koselleck (1975), p. 680 sqq.

119. WuG, p. 509, tout à fait comparable à la re-matérialisation du droit.

120. Benz (1995).

121. Hanke (2001), p. 33 sq. 
122. $M W G I / 17$, p. 170 sq.

123. GPS, p. 334 sq. ; MWG I/15, p. 466 sq. [«Domination des fonctionnaires et leadership politique ", OP, p. 332].

124. GPS, p. 351 sq. ; MWG I/15, p. 486 sq. [« Publicité de l'administration », OP, p. 356 sq.].

125. WuG, p. 574, MWG I/22-4, p. 221 sq.

126. Fondamental sur ce point, Lepsius (1995), p. 328 sqq.

127. Mommsen (1974), p. 163 sqq., 387 sqq. ; GPS, p. 352 sq. et 357 [OP, p. 356 sq.].

128. GPS, p. 331 ; MWG I/15, p. 463 [OP, p. 307 sq. et 334]. Selon Weber, « compte tenu de la nature de ses forces motrices idéelles et matérielles, et de la nature de la vie économique actuelle, [que] sa défaillance mènerait à la catastrophe, la machinerie bureaucratique serait prête, le cas échéant [...] à servir les yeux fermés quiconque se trouverait en possession des moyens de pouvoir nécessaire et garantirait aux fonctionnaires le maintien dans leurs postes. » (GPS, p. 451 ; MWG I/16, p. 103).

129. WuG, p. 569 ; MWG I/22-4, p. 208.

130. On trouve un aperçu des études classiques sur cette thématique dans Mayntz (1997), p. 64 sqq.

131. Schnapp (2004). Sur la critique de Schnapp, voir Die Öffentliche Verwaltung, 23 (2005), p. 970 sqq.

132. Formant école : Pressman / Wildavsky (1973) ; Hood (1976). Entre autres : Bohne (1981) et Delley et al. (1984). L'une des rares études sur la transposition des directives sociales européennes a été présentée par Treib (2004), qui montre entre autres qu'ici, ce sont moins la compatibilité ou l'incompatibilité entre les modèles européens et les traditions de régulation nationales qui jouent un rôle, que l'influence des partis politiques ou des constellations partisanes.

133. Sur les détails, voir Schluchter (1980), p. 159 sqq.

134. Constitution du Reich en date du 16 avril 1871. La clause stipulait qu'un membre du gouvernement ne pouvait pas être membre du parlement.

135. Lang (2005), p. 222 et 222-225.

136. Lang (2005), p. 229 et 229 sq.

137. Derlien / Lang (2005), p. 116.

138. Dogan (1989), p. 5, cité d'après Derlien / Lang (2005), p. 116, note 10.

139. Derlien / Lang (2005), p. 116, note 12 : «Sur les 23 secrétaires d'État entre 1871 et 1890, 21 étaient issus du service de l'État prussien et 18 d'entre eux avaient occupé immédiatement avant leur nomination des postes de hauts fonctionnaires ministériels. En 1910, 6 des 7 secrétaires d'État du Reich étaient des fonctionnaires promus. Parmi les 7 successeurs de Bismarck à la fonction de chancelier du Reich, quatre étaient des bureaucrates expérimentés. » Voir également Morsey (1957), p. 251.

140. Lang (2005), p. 223-225, notamment p. 229 sq.

141. Derlien (1991), p. 173. (Base de données : tous les ministres fédéraux et chanceliers entre 1949 et 1984, tous les secrétaires d'État parlementaires entre 1967 et 1984).

142. La parlementarisation ne signifie pas seulement le renforcement du parlement, mais aussi celui des partis politiques. Sur la typologie wébérienne des partis, WuG, p. 167 sqq. [E\&S, p. 371 sq.] ; également Breuer (1998), p. 199 sqq., ainsi que Breuer (2003), notamment p. 353-359.

143. Mommsen (1974), p. 437.

144. Mommsen (2001), p. 318.

145. Breuer (1994), p. 145 ; également Breuer (1994a).

146. Entre autres, Budäus (1998), p. 1 sq. ; Reichard (1995), qui caractérise cependant le modèle de bureaucratie de Weber d'une manière différente et contradictoire. - Au lieu de « direction bureaucratique », on devrait parler de « direction légale » (c'est-à-dire de direction par le droit et la loi), même si Weber n'était pas intéressé par cette thématique. 
147. Que le niveau visé soit effectivement celui de la philosophie de la réforme apparaît dans le bilan des réformes NPM dressé un peu plus tard par Reichard (2002). Kuhlmann ([2004], p. 376 sqq.) dresse un bilan lui aussi dégrisant de la mise en œuvre du Nouveau Modèle de Direction (la variante allemande de la réforme NPM) par les communes. Un inventaire international comparé a été présenté par Pollit / Bouckaert (2004). Pour ce qui concerne la bureaucratie ministérielle de la RFA, citons Derlien (2003a), p. 114 : «In 1990, managerialism was hardly visible in Germany. German bureaucracy, despite thirty years of constant structural adjustment, had stayed basically Weberian. The concept of legal-rational polity with a bureaucratic staff had remained dominant [...].» On ne peut toutefois pas en conclure qu'une «direction hiérarchique » (par transmission d'instructions) caractérise le mode de travail ; «la relation entre administration ministérielle et direction politique » se présente plutôt " comme une sorte de dialogue [...] permanent. » (Mayntz [1997], p. 193).

148. Lepsius (1995a).

149. Lepsius (1995), p. 327.

150. Lepsius (1995), p. 328.

151. Lepsius (1995), p. 332.

152. Lepsius (2000), p. 290, 290 sqq. Un tel groupement de domination s'exprime, dans le quotidien, sous la forme d'une administration et s'efforce, comme toute domination installée sur la durée, de se légitimer vis-à-vis des dominés, comme le montrent les épisodes qui se sont déroulés autour de la constitution européenne.

153. Bach (2005), p. 576, 601 sq. ; Bach (1999) p. 34, 88 sqq.

154. Par comparaison avec la part toujours relativement élevée de juristes dans la bureaucratie ministérielle allemande (Derlien [2003] p.406), la proportion de juristes dans ce corps de fonctionnaires est relativement faible (Bach [2005], p. 589 sq.).

155. On trouve des données quantitatives, variables selon le mode de décompte, chez Töller (1999), p. 333 sq. (en 1998 : quatre cents commissions de comitologie), ainsi que chez Bach (1995), p. 382 sq. et Bach (2005), p. 599 (au milieu des années 1990 : huit cents commissions et groupes d'experts; quatre cents commissions de comitologie). Ces différences de mode de comptage dépendent d'une conception «large " ou "étroite» de la «comitologie »: dans un cas, on embrasse la totalité du système des commissions de l'Union Européenne, dans l'autre on ne compte que ces commissions définies du point de vue formel et juridique (commissions de comitologie) « qui ont été mises en place à l'initiative du conseil lors du transfert à la Commission d'autorisations de mise en œuvre" (Bach [2005], p.597, note 85). S'il est difficile d'avoir un aperçu précis, c'est aussi que les commissions sont mises en place, puis dissoutes, en fonction des besoins. Sur le système des commissions de l'Union Européenne, voir d'autre part Falke (2000) ainsi que Bücker / Schlacke (2000).

156. Wessels (2000), p. 123, 195sq.

157. Bach (2005), p. 601. Sur le mode de travail de cette « bureaucratie parallèle », voir Puntscher Riekmann (1998), p. 157 sq.

158. Puntscher Riekmann (1998), p. 85 sqq. Pour Puntscher Riekmann (1998), il s'agit également d'un savoir de la domination, mais il occupe une position particulière dans la mesure où elle considère certes également l'Union Européenne comme un groupement de domination en genèse, mais dont elle analyse la formation à la lumière de cette "administration par commission» (Hintze [1981(1910)]) qui, sous la forme d'organisations préalables des bureaucraties d'État modernes, anima la formation de l'État à l'époque de l'absolutisme. Dans cette mesure, elle se réfère moins à Max Weber qu'à Foucault, Hintze et Carl Schmitt.

159. Puntscher Riekmann (2000), p. 137.

160. Bach (2005), p. 605.

161. Lepsius (2003), p. 33.

162. Lepsius (2003), p. 33. 
163. WL, p. 440 [ETS p. 347]; WuG, p. 6 sq. [E\&S, p. 41].

164. Mommsen (1965) p. 137 ; WuG, p. 13, § 3, alinéa 2 [E\&S, p. 58 sq.]. Voir aussi Anter (1996), p. 94 sq., qui cite lui aussi cette lettre de Max Weber. Traduction de la lettre par Jean-Pierre Grossein, in : Revue française de sociologie, 2005, 46-4, p. 923-928, ici p. 924.

165. WuG, p. 6 sq. [E\&S p. 39 sq.] ; Anter (1996), p. 101.

166. WL, p. 448 sq. [ETS, p. 358 sq].

167. Mayntz (1995).

INDEX

Mots-clés : État, bureaucratie, domination, institution, groupement, fonction publique, ordre, organisation

Schlüsselwörter : Staat, Bürokratie, Herrschaft, Anstalt, Verband, Beamtentum, Ordnung, Organisation

\section{AUTEURS}

\section{HUBERT TREIBER}

Hubert Treiber est professeur émérite de sciences administratives à l'Université Leibniz de Hanovre. Pour plus d'informations, voir la notice suivante. 